\title{
Physiology of Zinc Oxide Nanoparticles in Plants
}

\author{
Réka Szőllősi, Árpád Molnár, Gábor Feigl, Dóra Oláh, Márk Papp, \\ and Zsuzsanna Kolbert
}

\section{Introduction}

Zinc oxide $(\mathrm{ZnO})$ is a multifunctional material with unique physical and chemical properties, for example, broad range of radiation absorption, high chemical and photostability and high electrochemical coupling coefficient (Segets et al. 2009; Lou 1991). The covalence of $\mathrm{ZnO}$ is between ionic and covalent semiconductors and it is classified as a semiconductor in group II-VI. It has a high bond energy of $60 \mathrm{meV}$ and a broad energy band of $3.37 \mathrm{eV}$. The thermal and mechanical stability makes it useful in laser technology, electronics and optoelectronics (Bacaksiz et al. 2008; Wang et al. 2005). It has multiple uses in hydrogen production, ceramic industry, biomedicine, pro-ecological systems or plant disease management (Wang 2008; Chaari and Matoussi 2012; Özgür et al. 2005; Bhattacharyya and Gedanken 2007; Ludi and Niederberger 2013; Elmer et al. 2018). ZnO has three crystal structures in nanoparticles: wurtzite, zinc-blende and rock salt (Özgür et al. 2005; Moezzi et al. 2012). Similar to other metallic engineered nanoparticles, its size range is within 1-100 nm (Marslin et al. 2017). ZnO crystals can appear as 1 D, 2 D or 3 D structures with a large variety of morphology (Kołodziejczak-Radzimska and Jesionowski 2014), which affects the toxicity and influences of the nanoparticles (Stanković et al. 2013). It was estimated that nearly 30,000 tons of ZnO NPs is used per year in various products, such as textiles, pigments, semiconductors, industrial coatings, medicines, food additives and sunscreens (Mukherjee et al. 2016; Mishra et al. 2017; The Global Market for Metal and Metal Oxide Nanoparticles 2010-2027). $\mathrm{ZnO}$ NPs are often used as a nanofertiliser; however, they can increase the $\mathrm{Zn}$ ion levels in the soil in excess of expected concentrations (Watson et al. 2015).

Many factors have an impact on the exact outcome of the ZnO NP-plant interactions, including the investigated plant species, the size of the applied particles, the

R. Szőllősi (四) · Á. Molnár · G. Feigl · D. Oláh · M. Papp · Z. Kolbert Department of Plant Biology, University of Szeged, Szeged, Hungary e-mail: szoszo@bio.u-szeged.hu 
duration or existence of pre-cultivation, the concentration and duration of $\mathrm{ZnO}$ exposure or the applied growth conditions, namely germination test in Petri dishes or hydroponics or pot experiment. Up to now, it has been well reviewed that how the metallic nanoparticles (including ZnO NPs) may influence the development, the photosynthetic activity or other processes there is still much lack of our knowledge (Marslin et al. 2017; Hou et al. 2018; Pullagurala et al. 2018b).

\section{The Uptake and Transport of ZnO NPs in Higher Plants}

The uptake and accumulation of ZnO NPs is not fully understood up to this date, but it consists of two major pathways: zinc ion release and direct nanoparticle accumulation (Poynton et al. 2011). Zinc homeostasis is regulated in plants through transporter proteins, which control the intake, mobilisation and compartmentalisation of the ion (Clemens 2001). Well-known zinc transporter protein families are as follows: ZIP (ZRT, zinc transporter proteins; IRT-like protein) are tasked with zinc uptake in the root system, root to shoot translocation is realised via HMA (heavy metal ATPases) proteins, and MTP (metal tolerance protein) is used for compartmentalisation and detoxification (Pence et al. 2000). The uptake and translocation of $\mathrm{ZnO}$ NPs is much less investigated. In soil, the interactions between soil grain, clay minerals and nanoparticles determine the transport, the fate and the behaviour of nanoparticles (Darlington et al. 2009). García-Gómez et al. (2018b) presented in case of several vegetables and crops that $\mathrm{pH}$ values or other characteristics of the soil may determine the impact of ZnO NPs on plants (Table 1c). ZnO NPs are absorbed on kaolin surfaces, followed by a dissolution (Scheckel et al. 2010). Accumulation of $\mathrm{ZnO}$ NPs on root surface areas is supported by multiple sources. Lin and Xing (2008) detected large amounts of nanoparticles adhered to the root epidermis in ryegrass applying scanning electron microscopy. In Schoenoplectus tabernaemontani $\mathrm{ZnO}$ NPs were observed on the root surface (Zhang et al. 2015), as well as in case of maize roots where nanoparticles were absorbed on the surface (Lv et al. 2015). The accumulation of zinc was examined in $\mathrm{ZnO}$ NP-treated sweet potato tubers and large amounts of $\mathrm{Zn}$ accumulated in the outer layers (namely the peel) of the tubers, which could have been nanoparticles (Bradfield et al. 2017; Table 1d). There are some reports of $\mathrm{ZnO}$ NPs invading tissues or even cells in ryegrass (Lin and Xing 2008), onion (Kumari et al. 2011), maize (Zhao et al. 2012; Lv et al. 2015), rice (Chen et al. 2018a) and Schoenoplectus tabernaemontani (Zhang et al. 2015). Since plants in natural conditions usually grow in the soil, the root tissues and cells are the first targets of $\mathrm{ZnO} \mathrm{NP}$ "invasion", mainly at higher doses. The main symptoms of ZnO NP toxicity are reduced root length and consequently higher root diameter, sometimes fewer root hairs (Lee et al. 2013; Balážová et al. 2018; Table 1d). Some reports showed that ZnO NPs may be transported until the endodermis using both apoplastic and symplastic pathway then they can enter the vascular cylinder (Lin and Xing 2008; reviewed by Lee et al. 2013; Lv et al. 2015) but there is not much evidence of translocation to shoot as nanoparticles. Chen et al. 


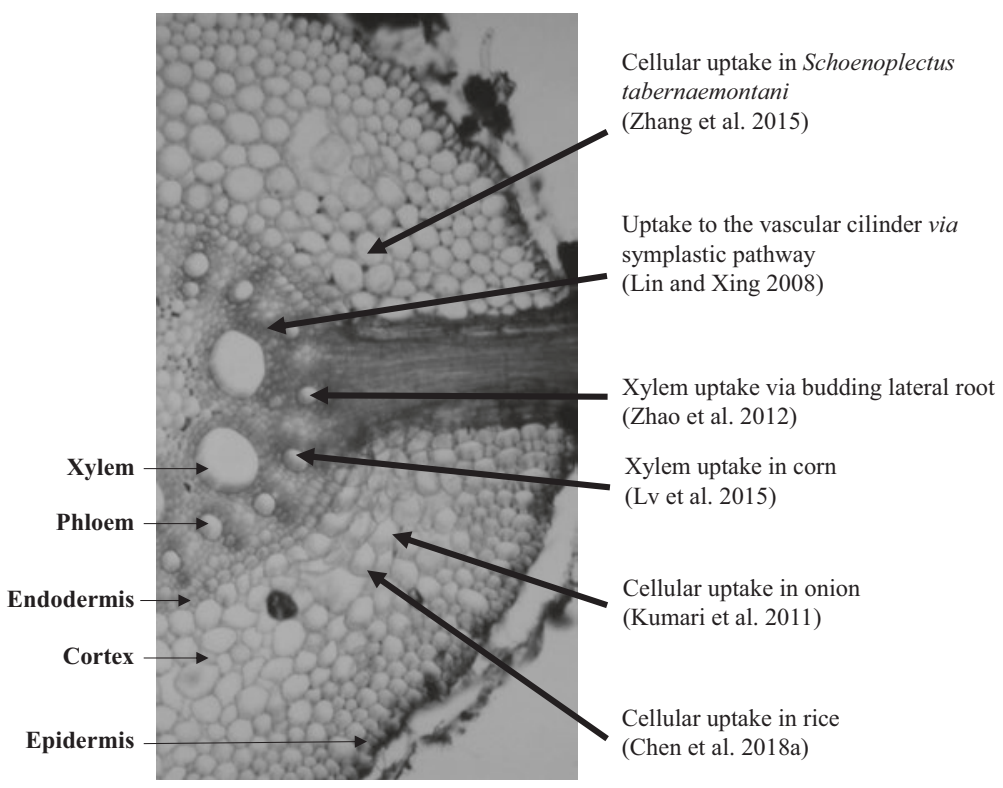

Fig. 1 Comparison of $\mathrm{ZnO} \mathrm{NP}$ uptake by different plant species at the tissue level

(2018a) demonstrated the presence of ZnO NPs as dark dots both in the intercellular space and in the cytoplasm of the root cortical cells in the elongation zone which supports the dual (symplastic and apoplastic) transport theory. Besides, it was exhibited that cell organelles can be also influenced, Lin and Xing (2008) detected $\mathrm{ZnO}$ NPs in the nuclei and cytoplasm, as well. The root uptake and the potential transport mechanisms of $\mathrm{ZnO}$ NPs are depicted in Fig. 1.

Raliya et al. (2015) detected ZnO NPs with TEM in the shoot and leaves of tomato plants but only after foliar application and not soil amendment. In Indian mustard, ZnO NPs were translocated to the leaves (Rao and Shekhawat 2014). At the same time, in soybean (López-Moreno et al. 2010) and mesquite roots (Hernandez-Viezcas et al. 2011), there were no detectable ZnO NPs, which indicates that nanoparticles entering the tissues is not a common phenomenon across all species.

It is well known that plant cell wall has pores that measure up to several nanometres (Carpita et al. 1979), which should filter out nanoparticles and prevent them from entering the cell. It has been reported that, in bacteria, ZnO NPs may increase the permeability generating "holes" in cell walls to reach the plasma membrane (Stoimenov et al. 2002; Brayner et al. 2006). Between cells, nanoparticles are most likely transported via plasmodesmata, which have a reported diameter of $\sim 40 \mathrm{~nm}$ (Tilney et al. 1991). To enter the cortex, there are two possible ways: (1) entering it through the plasmodesmata as previously mentioned, or (2) potentially entering it via budding lateral roots which temporarily allow nutrients to pass the Casparian strip (Bell et al. 2003; Lv et al. 2015). 
It seems that $\mathrm{ZnO}$ NPs may influence living cells via three distinct pathways: (1) biotransformation and release of Zn (II) ions, (2) surface interaction of nanoparticles resulting in harmful molecules such as reactive oxygen species (ROS) and (3) direct interaction of nanoparticles with cell metabolism, like photosynthesis and nutrient homeostasis (Brunner et al. 2006). ZnO NPs undergo biotransformation due to humic acid and other organic root exudates then they penetrate the root through the root pores and it is accompanied by the uptake processes, as it has been described in many studies and accumulate in tissues of plants, mainly in ionic form (Chen et al. 2018a; López-Moreno et al. 2010; Raliya et al. 2015; Balážová et al. 2018). In rice, Chen et al. (2018a) demonstrated that the plants can accelerate the degradation process of $\mathrm{ZnO}$ NPs, resulting in a higher $\mathrm{Zn}$ ion concentration. Similar results were obtained by $\mathrm{Lv}$ et al. (2015) in maize, proving the importance of this pathway. It is important to note that the effects of nanoparticles are more than just the release or effects of $\mathrm{Zn}$ ions, which has been described by numerous studies (Lin and Xing 2008; Chen et al. 2018a; Poynton et al. 2011; Zhang et al. 2015; Bradfield et al. 2017). $\mathrm{Zn}$ accumulation triggered by $\mathrm{ZnO} \mathrm{NP}$ treatment has a lower translocation factor to shoot when compared to direct $\mathrm{Zn}$ ion treatment in cilantro (Pullagurala et al. 2018a, b; Table 1a), ryegrass (Lin and Xing 2008), Schoenoplectus tabernaemontani (Zhang et al. 2015), unlike previous examples in maize (Zhao et al. 2012) translocation factors were between 0.8 and 2 .

\section{ZnO NPs and Oxidative Stress}

Metal oxide nanoparticles have distinct antimicrobial properties, which are well examined (Sirelkhatim et al. 2015), and one of the proposed mechanisms is the generation of ROS (Huang et al. 2008; Xia et al. 2008; Lipovsky et al. 2009). ZnO NPs will produce ROS under visible or UV light, like superoxide anion or hydrogen peroxide (Sawai et al. 1998; Padmavathy and Vijayaraghavan 2008; Zhang et al. 2008; Jalal et al. 2010) and there are even reports of ROS generation in darkness, as well (Zhou et al. 2008; Adams et al. 2015). Since the electronic band structure of $\mathrm{ZnO}$ immediately absorbs photons with greater energy than $3.3 \mathrm{eV}$ and as a result $\mathrm{h}^{+}$positive holes and free electrons in conduction band are created (Seven et al. 2004). This positive hole is a strong oxidant and it will create reactive hydroxyl radicals (Zhang et al. 2012). It is also documented that nanoparticles can enhance ROS generation in plants (Wang et al. 2014; Barhoumi et al. 2015). The effect on $\mathrm{ZnO}$ NPs on the homeostasis of ROS seems to be dose dependent, as described by Javed et al. 2017, where lower (0.1, 1.0 and $10 \mathrm{mg} / \mathrm{L}) \mathrm{ZnO}$ concentrations had beneficial effects in Stevia plants such as increased antioxidant activity, but in contrast, at higher doses $\mathrm{ZnO}$ had toxic effect due to oxidative burst (Table 1a).

Positively, $\mathrm{ZnO}$ can stimulate the enzymatic antioxidants, e.g. superoxide dismutase (SOD), catalase (CAT) or peroxidase (POX), as it has been determined by Rizwan et al. (2019) (Table 1a), wherein treated wheat SOD and POX activities increased compared to control, similarly, in cotton lipid peroxidation (LP) decreased 


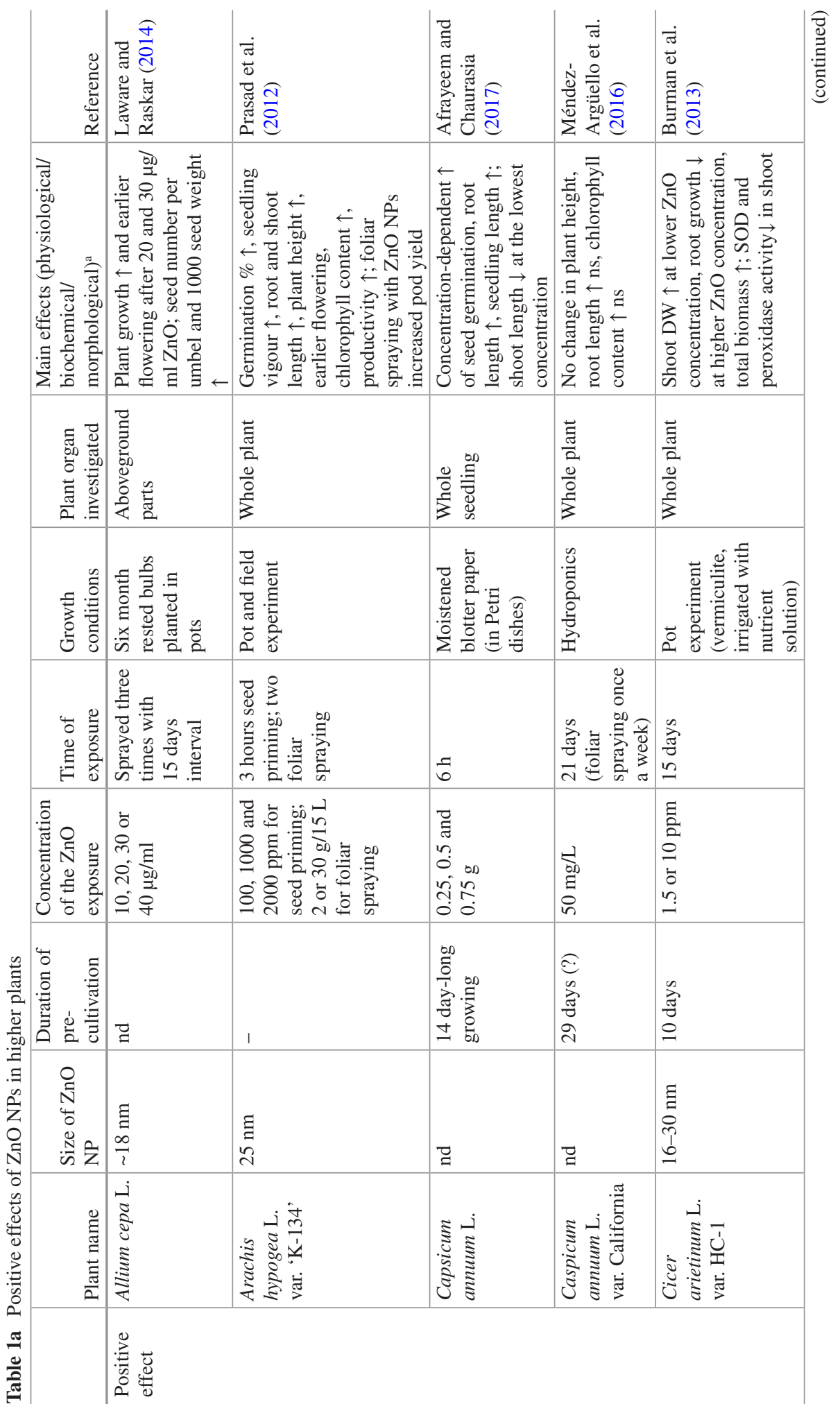




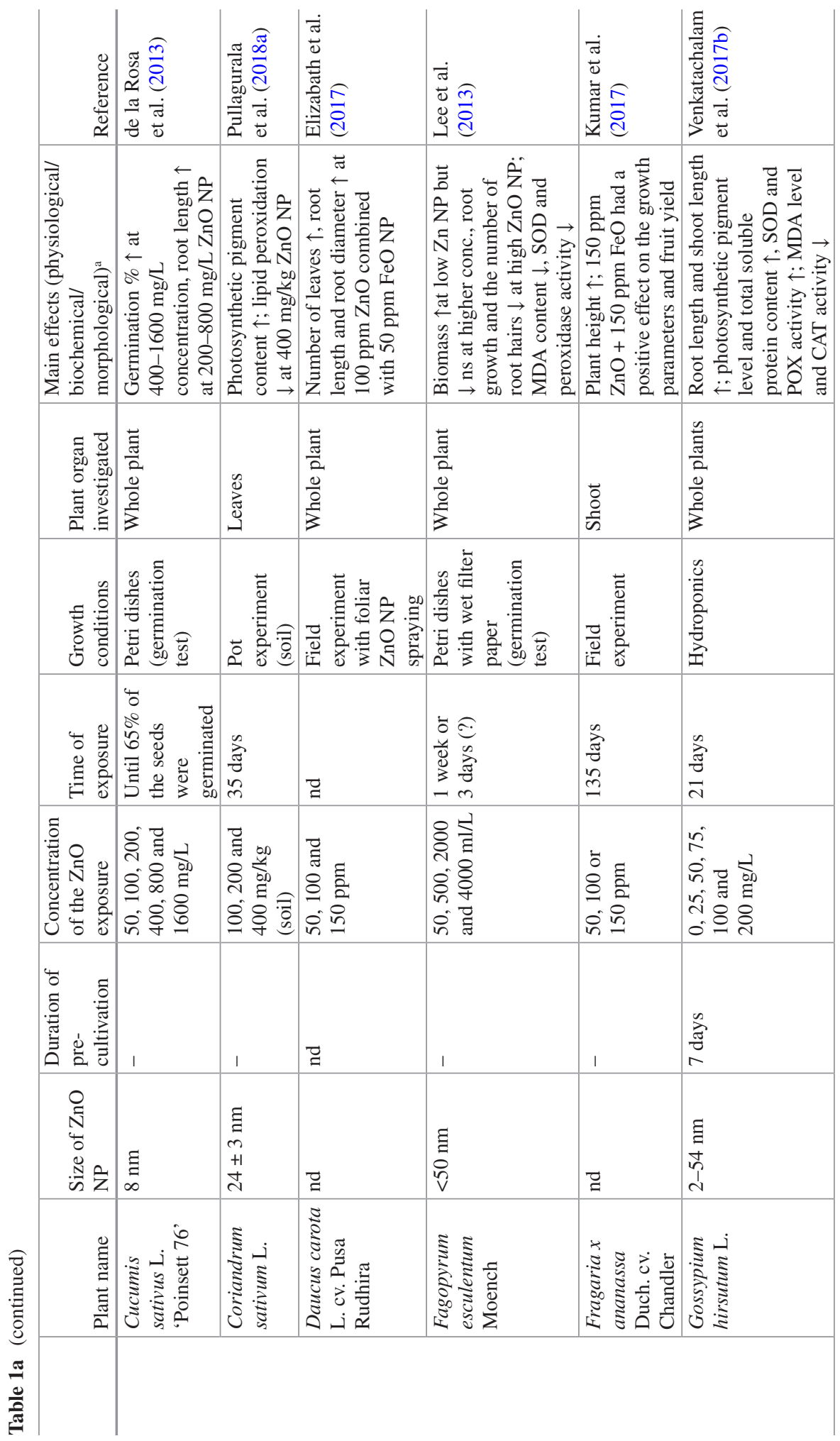




\begin{tabular}{|c|c|c|c|c|c|}
\hline 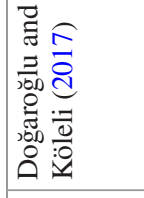 & 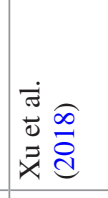 & 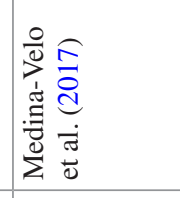 & 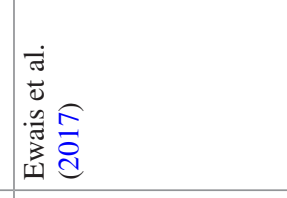 & 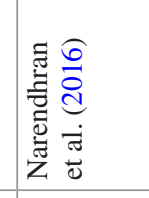 & 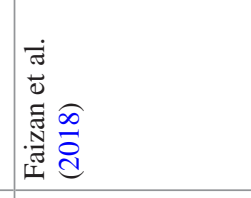 \\
\hline 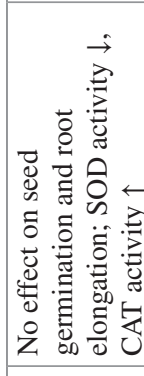 & 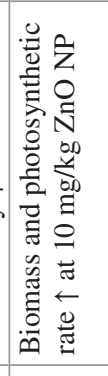 & 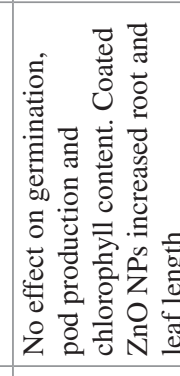 & 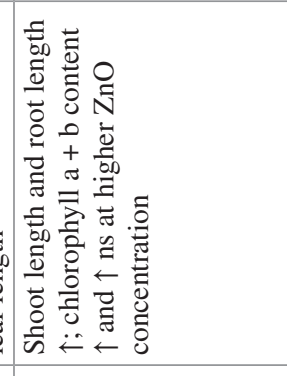 & 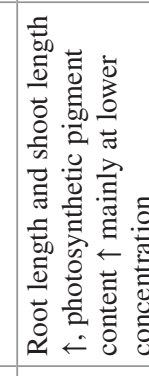 & 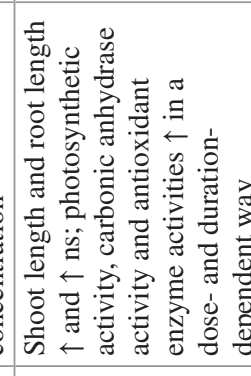 \\
\hline 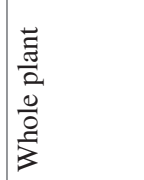 & 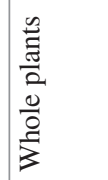 & 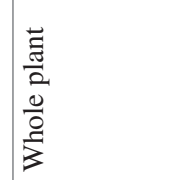 & 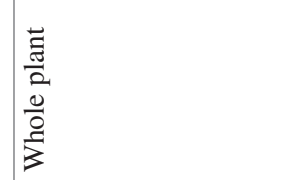 & 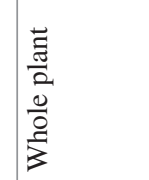 & 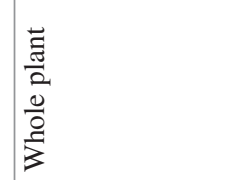 \\
\hline 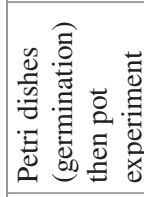 & 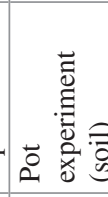 & 蒿 & 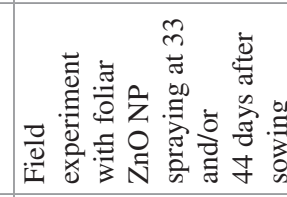 & 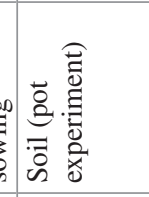 & 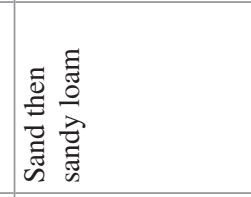 \\
\hline 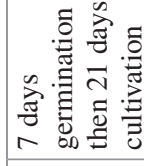 & 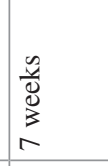 & 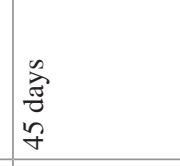 & $\Xi$ & 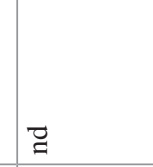 & 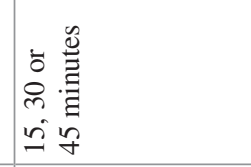 \\
\hline 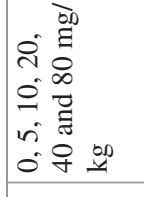 & 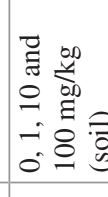 & 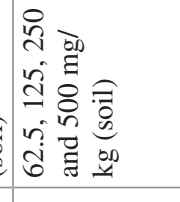 & 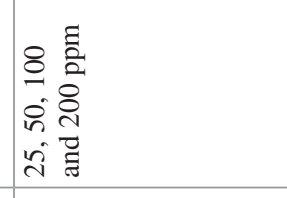 & $\begin{array}{l}0 \\
0\end{array}$ & 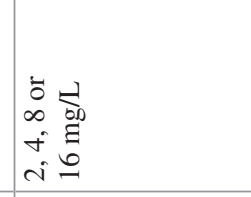 \\
\hline 1 & 1 & 1 & 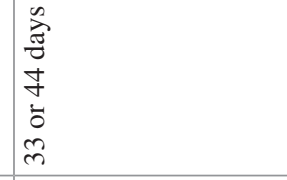 & 1 & 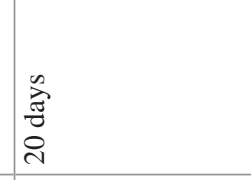 \\
\hline 志 & $\begin{array}{l}\vdots \\
\vdots \\
0 \\
+1 \\
0 \\
8\end{array}$ & 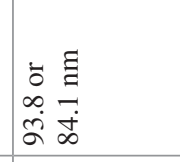 & $\Xi$ & 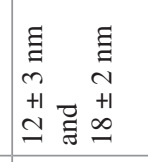 & 志 \\
\hline 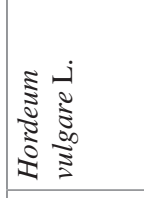 & نـ & 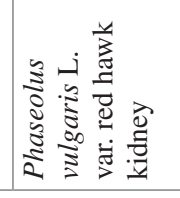 & 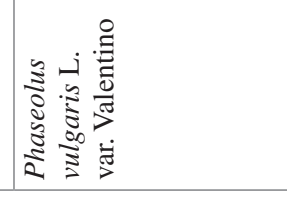 & 訔意 & 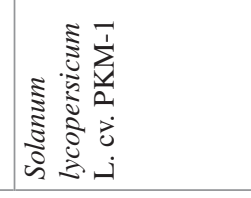 \\
\hline
\end{tabular}




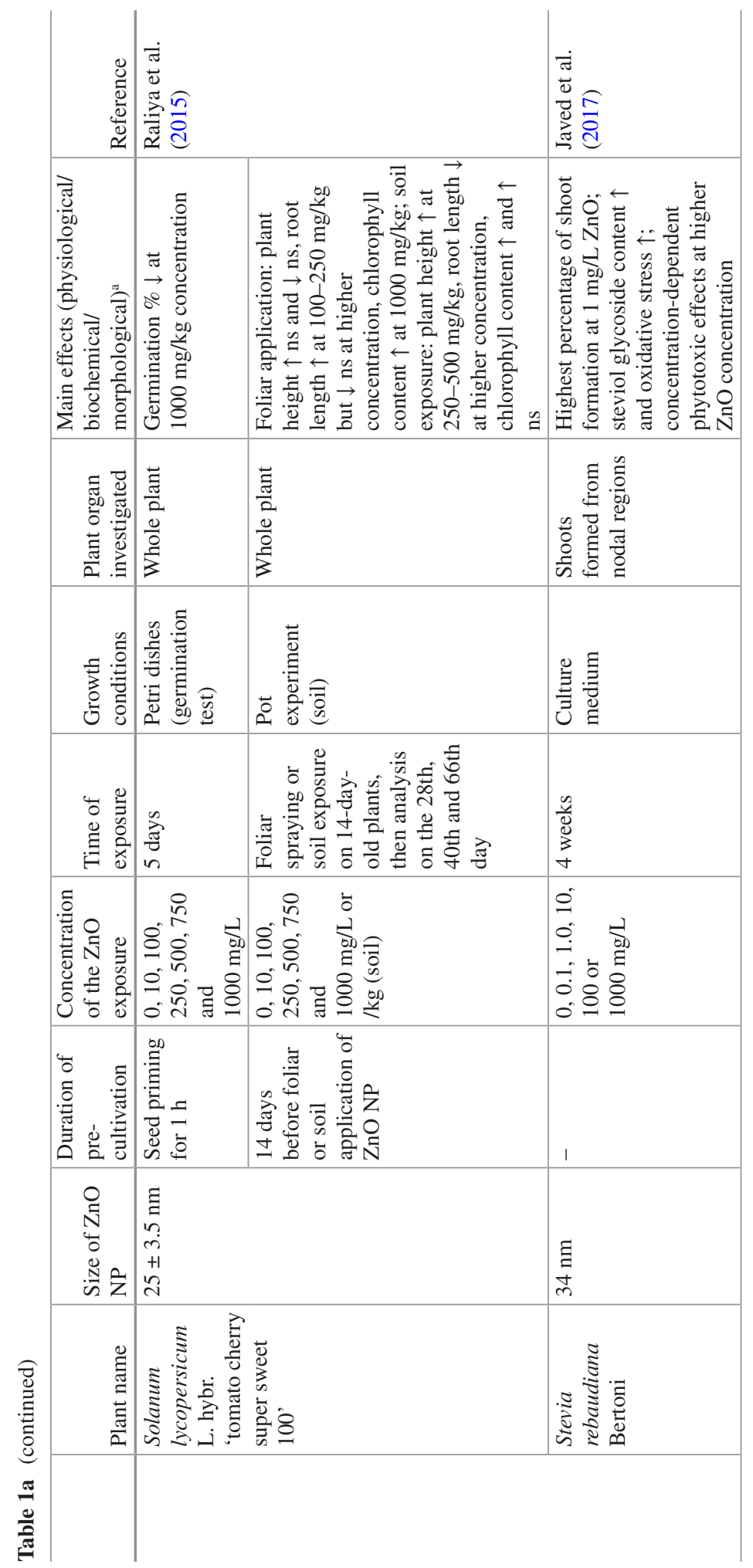




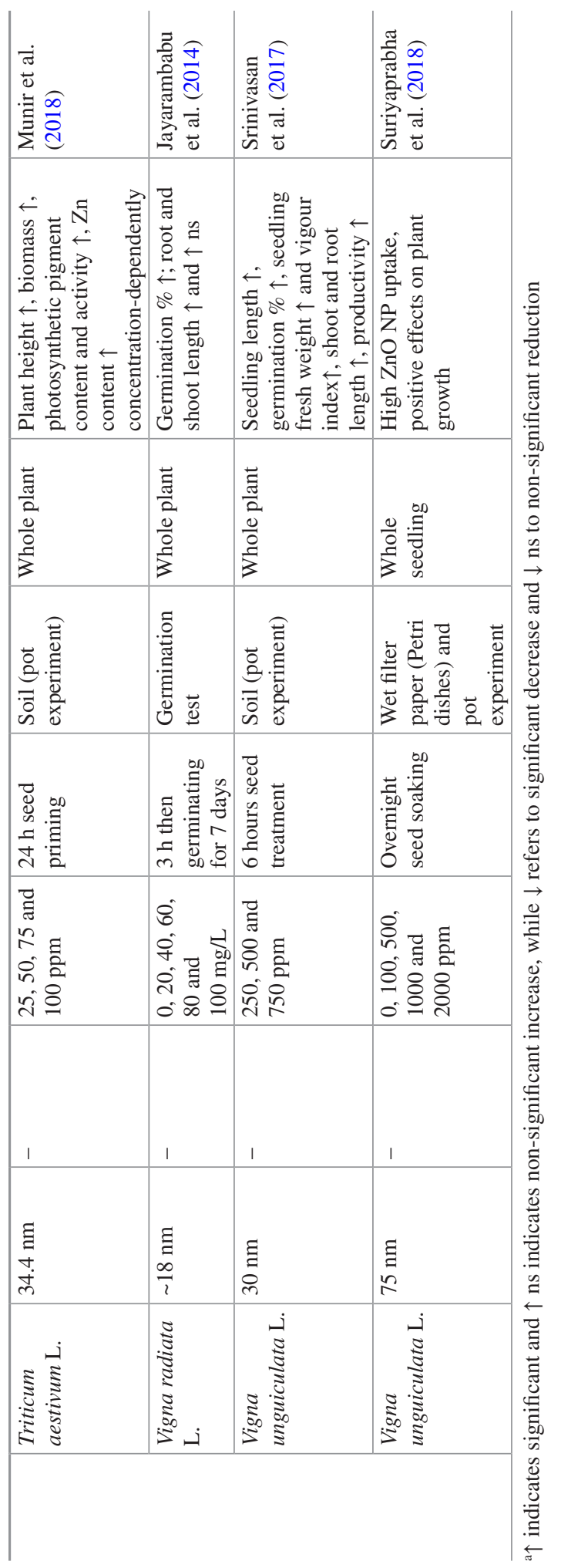




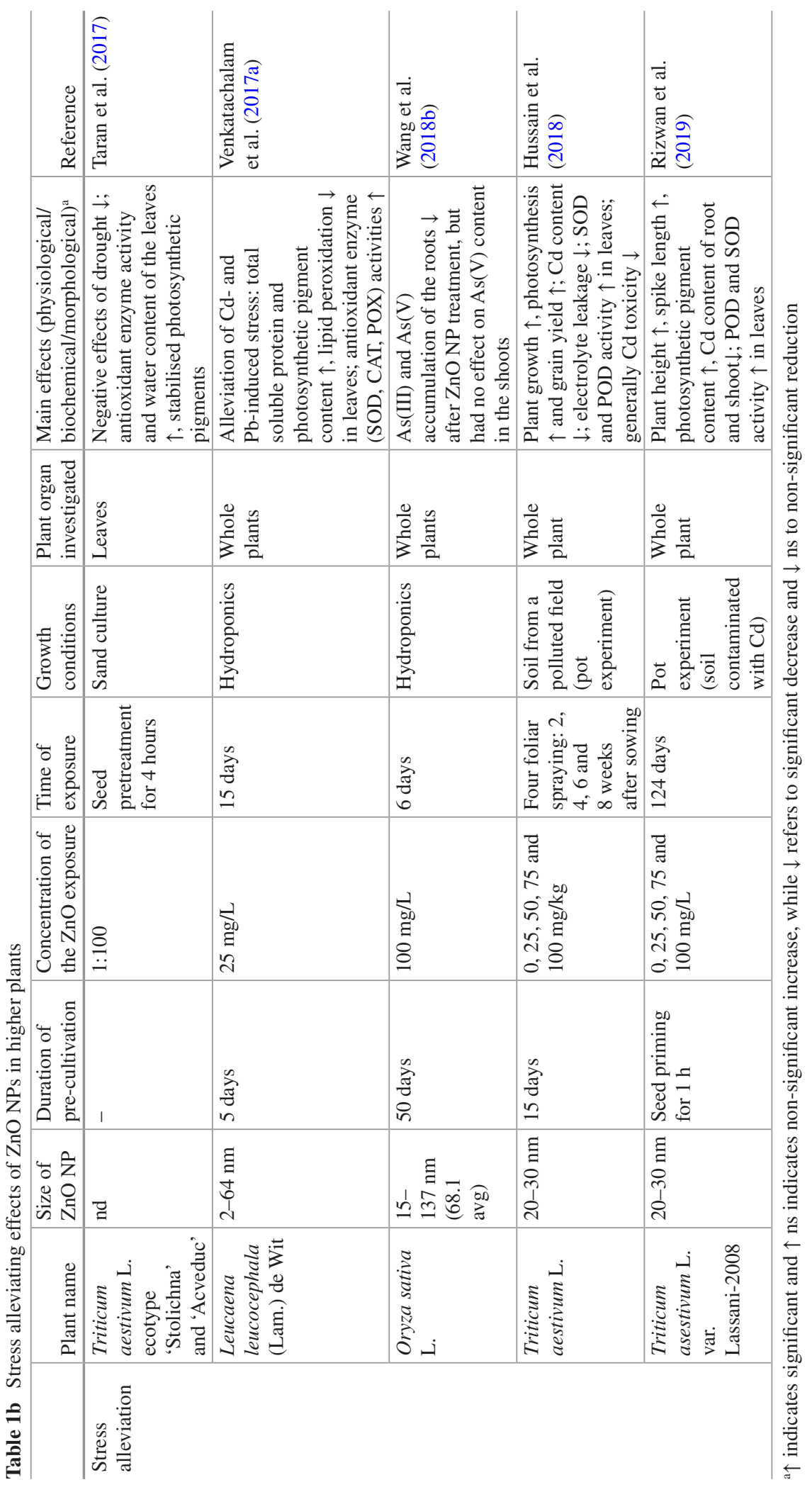




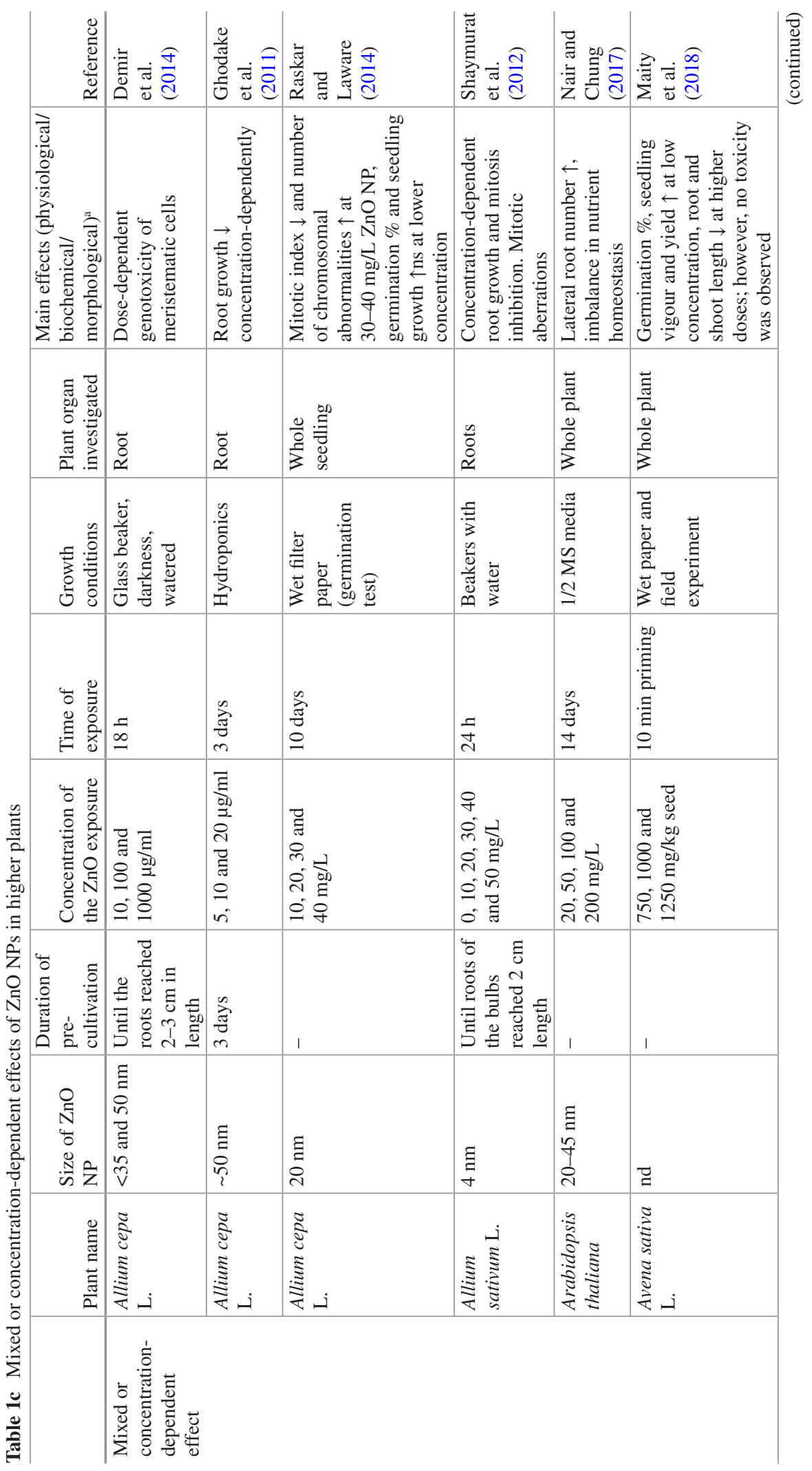




\begin{tabular}{|c|c|c|c|c|c|}
\hline 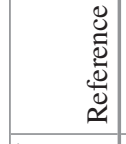 & 产 & 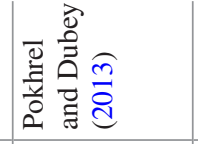 & 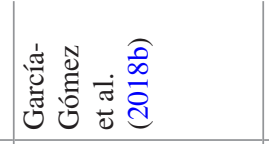 & 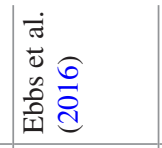 & 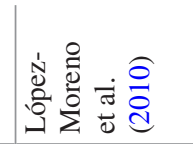 \\
\hline 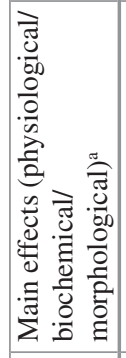 & 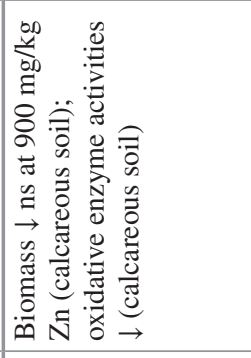 & 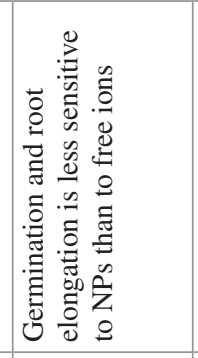 & 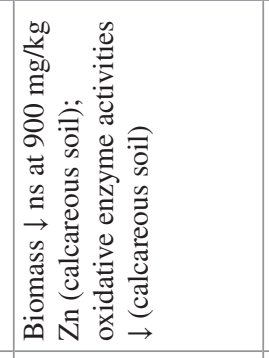 & 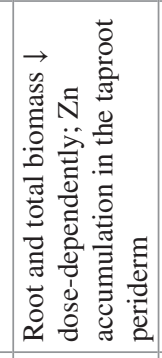 & 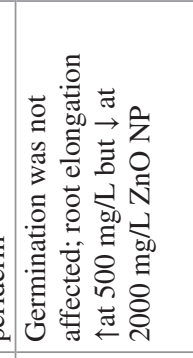 \\
\hline 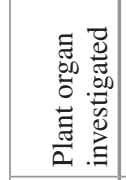 & 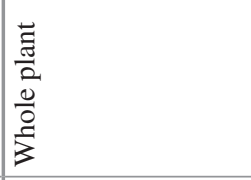 & $\begin{array}{l}\vec{\circ} \\
\stackrel{\leftrightarrow}{~}\end{array}$ & 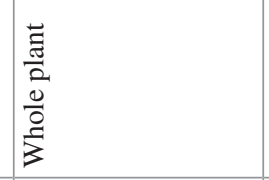 & 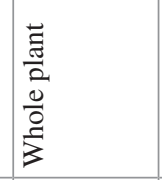 & 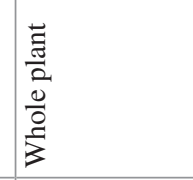 \\
\hline 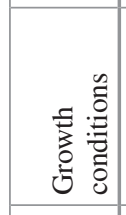 & 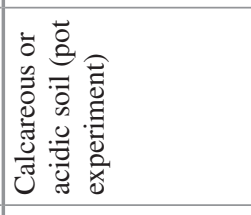 & 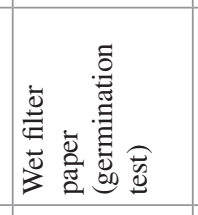 & 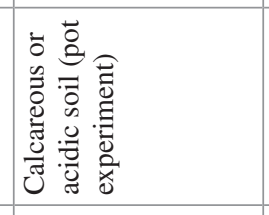 & 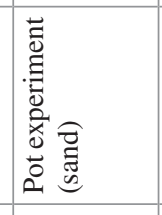 & 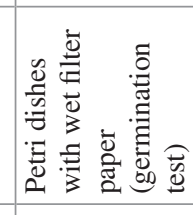 \\
\hline 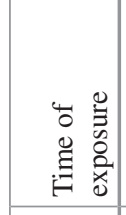 & 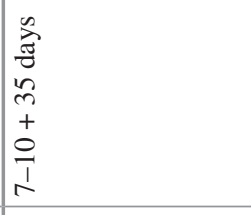 & 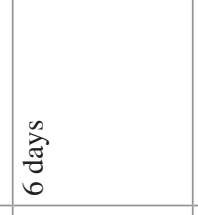 & $\begin{array}{l}0 \\
0 \\
\overrightarrow{3} \\
0 \\
0 \\
+ \\
0 \\
1 \\
1\end{array}$ & 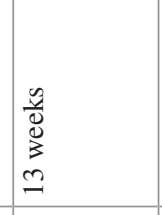 & 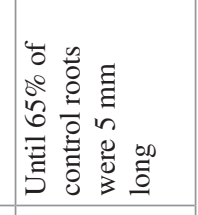 \\
\hline 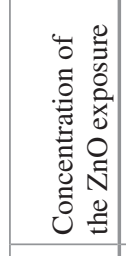 & 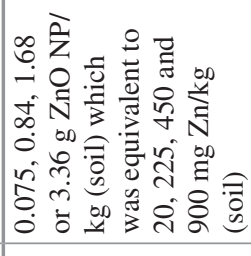 & 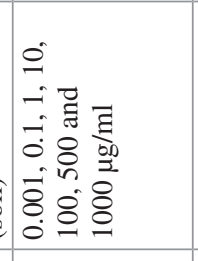 & 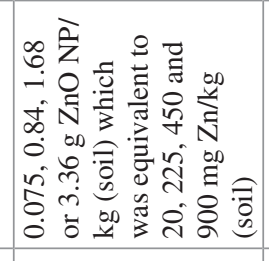 & 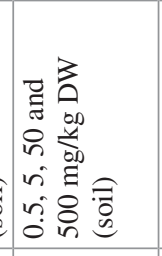 & 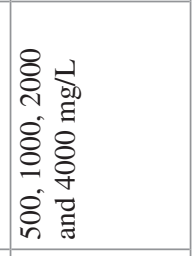 \\
\hline 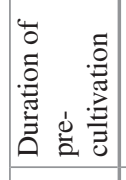 & , & 1 & 1 & 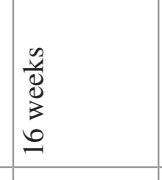 & 1 \\
\hline 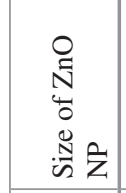 & $\begin{array}{l}\frac{E}{\bar{g}} \\
\frac{8}{\mathrm{v}}\end{array}$ & 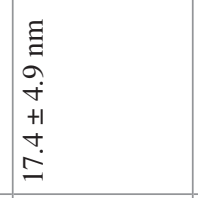 & 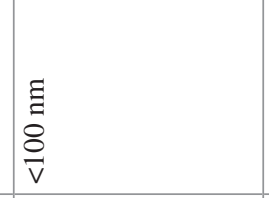 & $\begin{array}{l}\text { E } \\
\vdots \\
0 \\
0 \\
0 \\
\text { m }\end{array}$ & $\underset{\infty}{\underbrace{}_{\infty}}$ \\
\hline 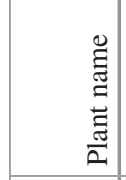 & 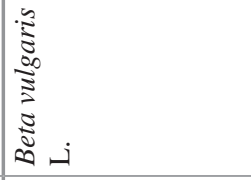 & 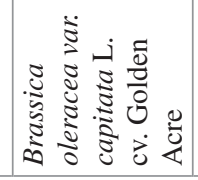 & 童 & 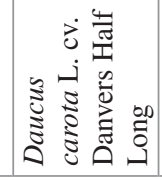 & 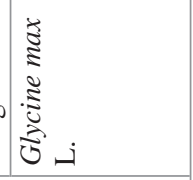 \\
\hline
\end{tabular}




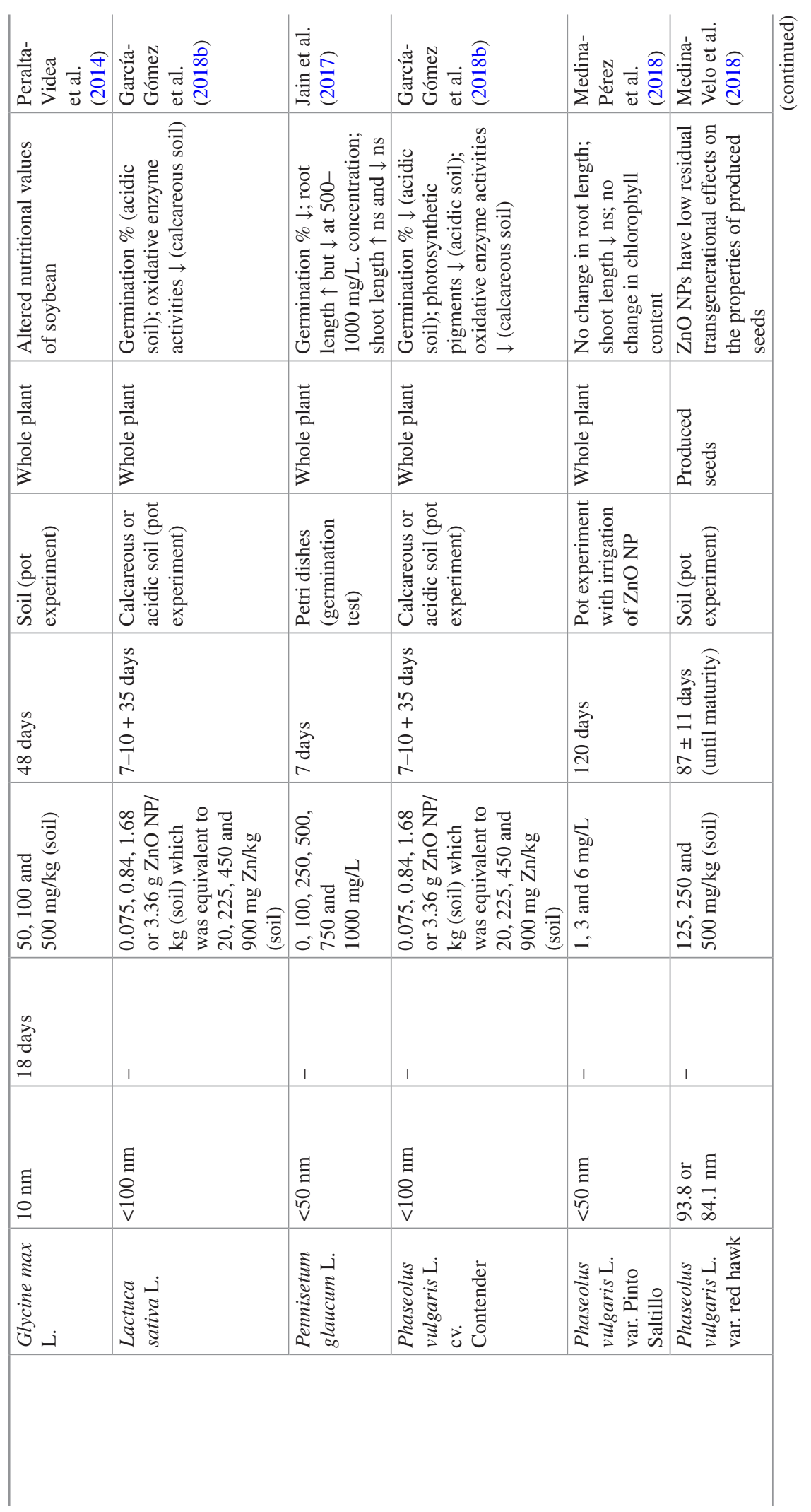




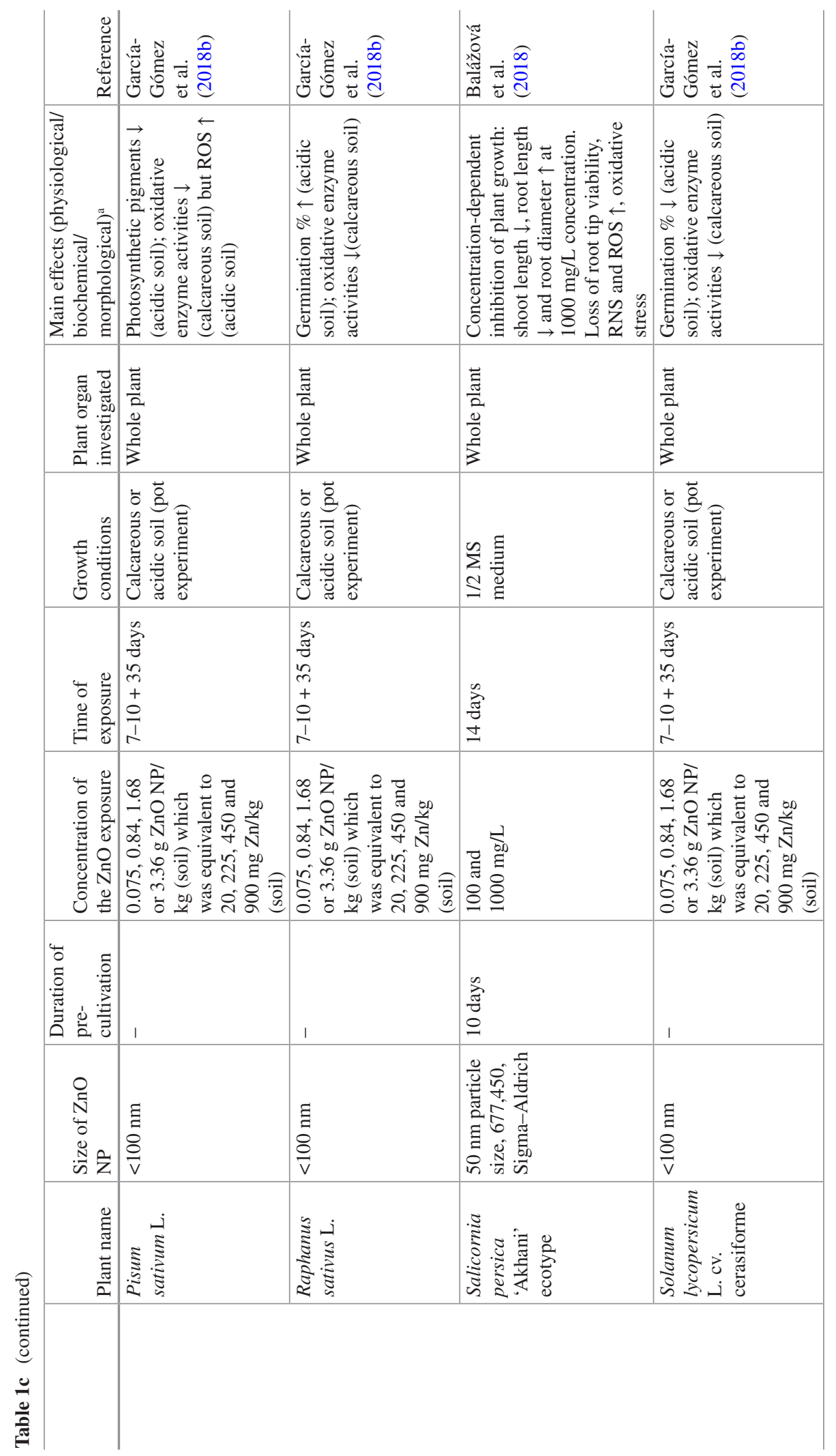




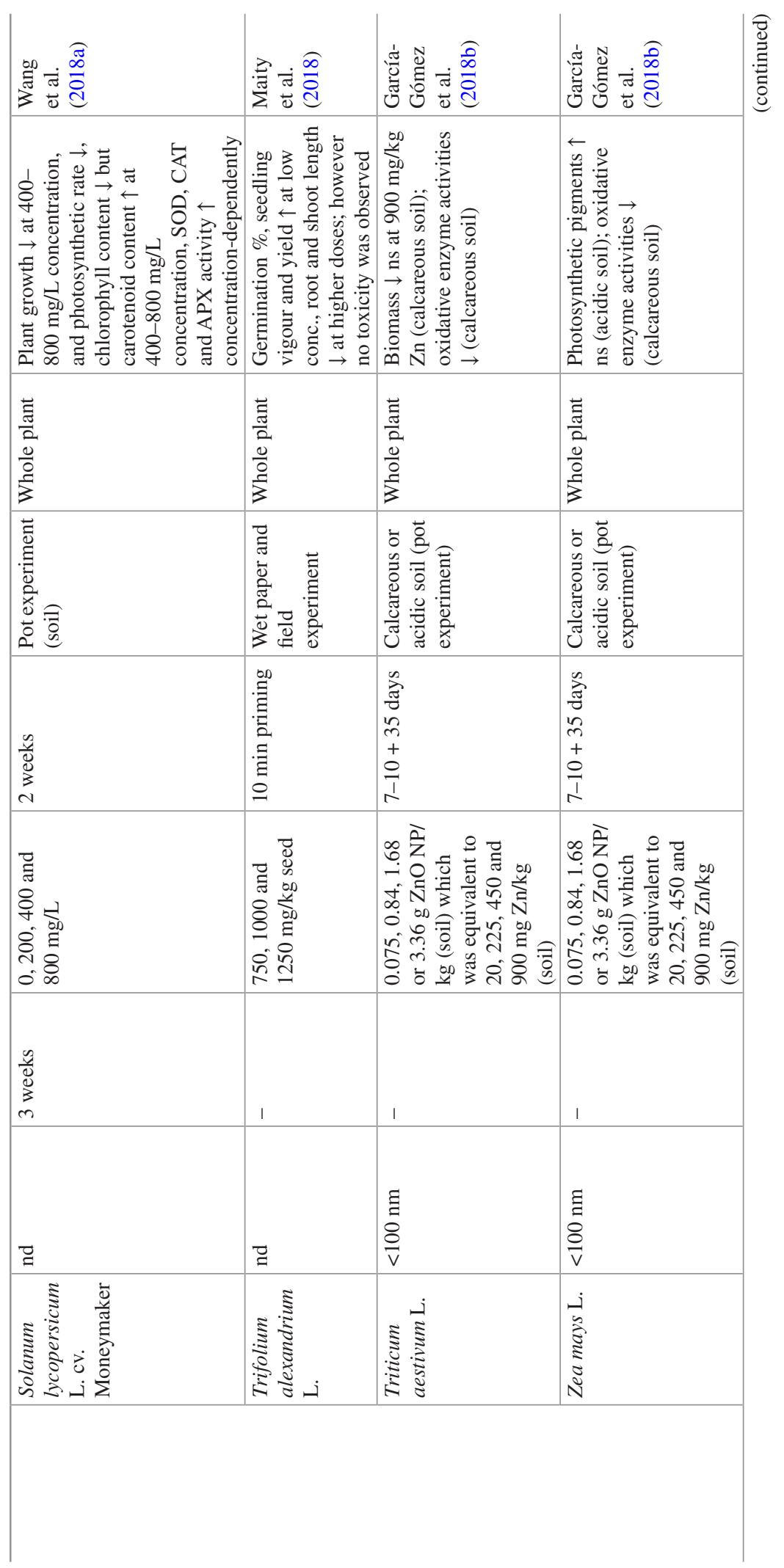




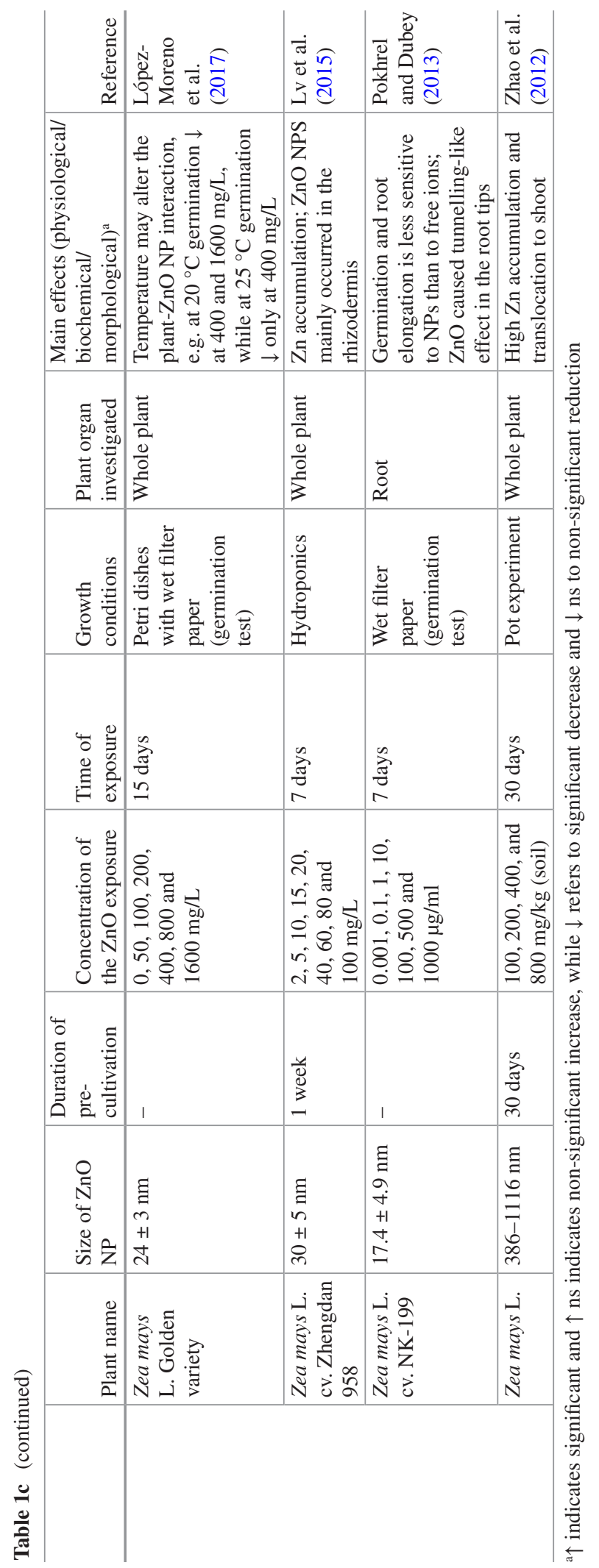




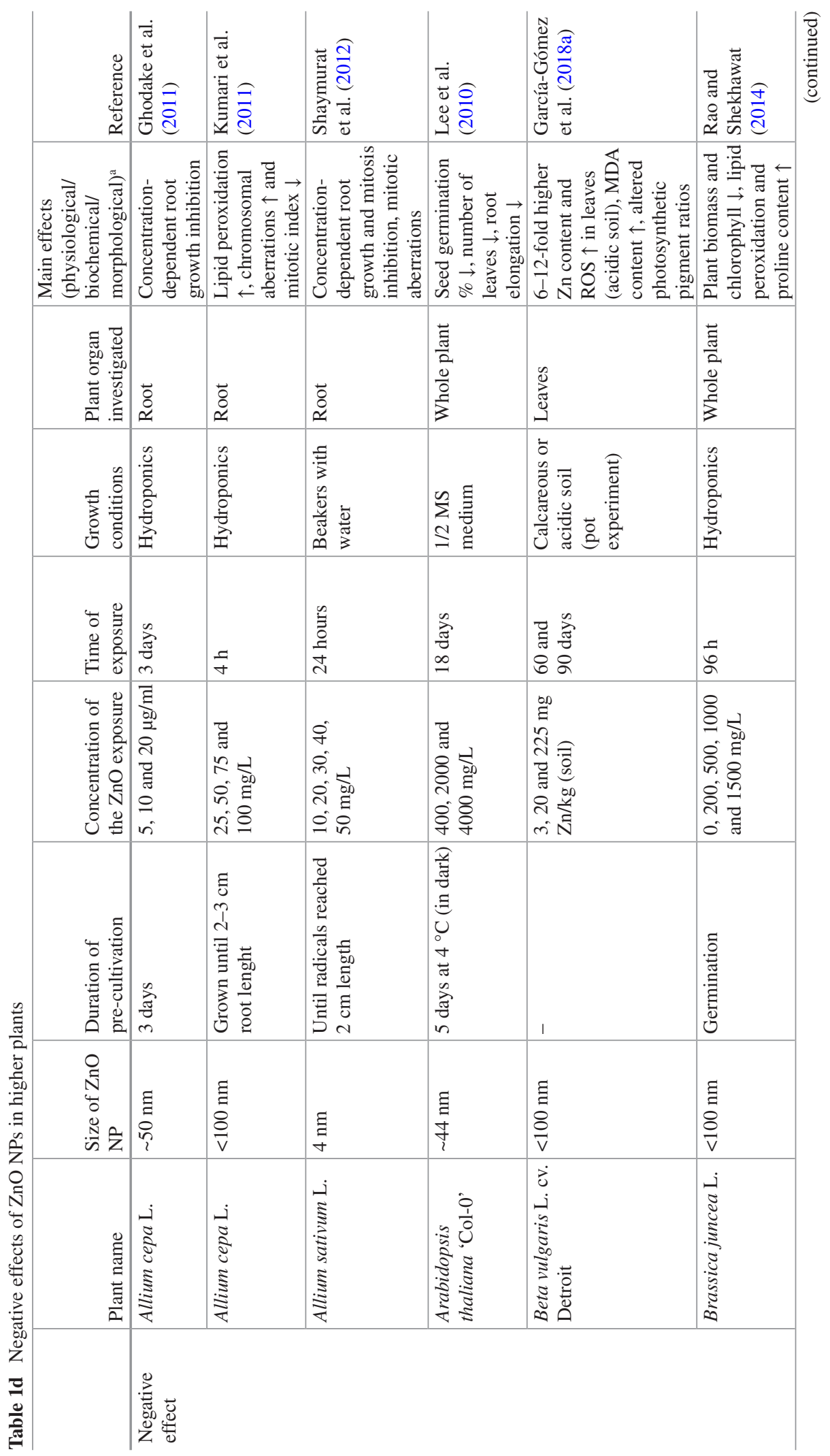




\begin{tabular}{|c|c|c|c|c|c|c|}
\hline 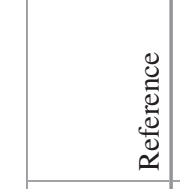 & 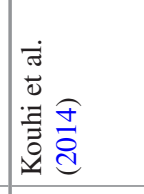 & 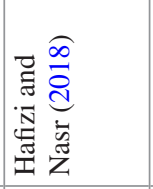 & $\begin{array}{l}\text { 离 } \\
\text { 过 } \\
\text { 言 }\end{array}$ & 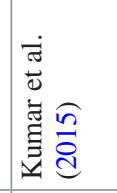 & 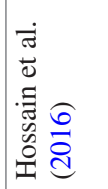 & 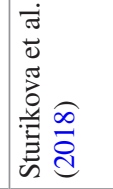 \\
\hline 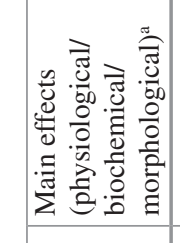 & 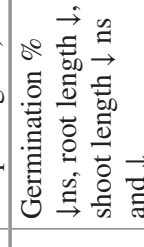 & 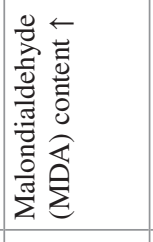 & 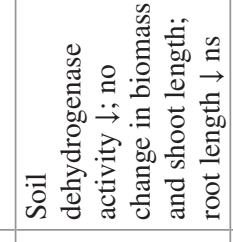 & 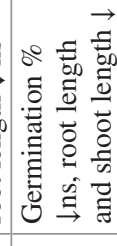 & 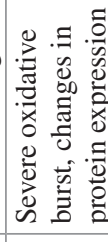 & 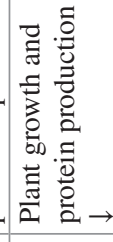 \\
\hline 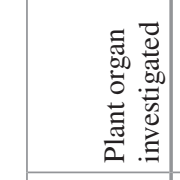 & 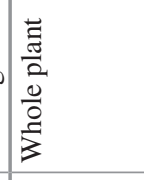 & 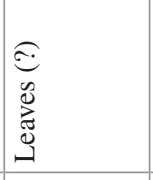 & $\begin{array}{l}\frac{1}{5} \\
\frac{5}{2} \\
\frac{2}{0} \\
\frac{0}{3} \\
\frac{1}{3}\end{array}$ & 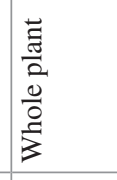 & $\begin{array}{l}\frac{5}{5} \\
\frac{5}{2} \\
\frac{0}{0} \\
\frac{0}{3} \\
\frac{1}{3}\end{array}$ & 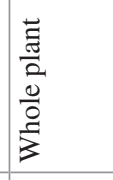 \\
\hline 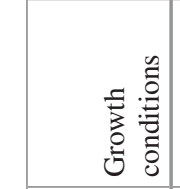 & 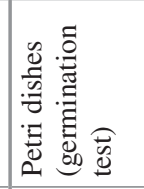 & 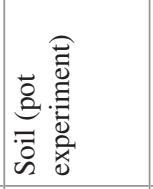 & 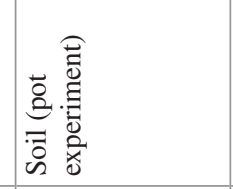 & 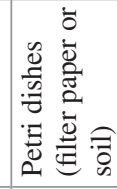 & 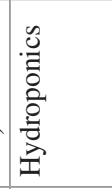 & 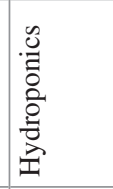 \\
\hline 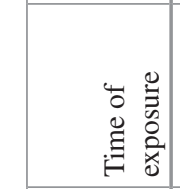 & 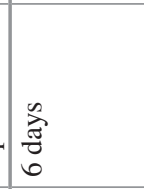 & 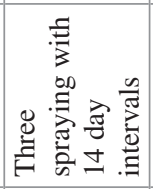 & 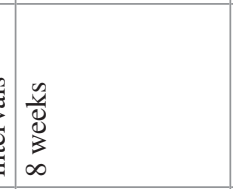 & 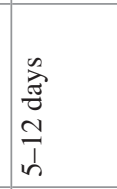 & 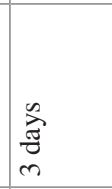 & 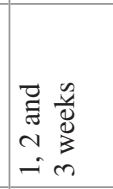 \\
\hline 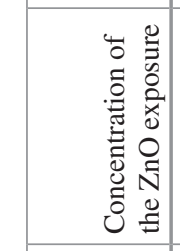 & 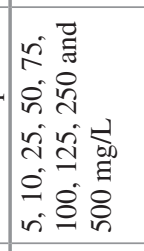 & 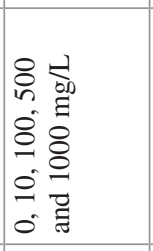 & 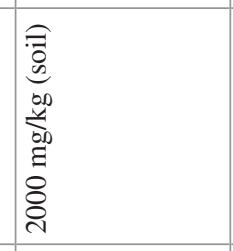 & 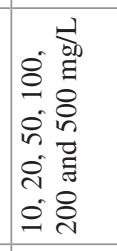 & 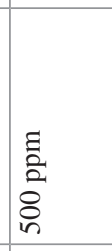 & 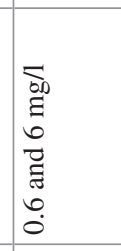 \\
\hline 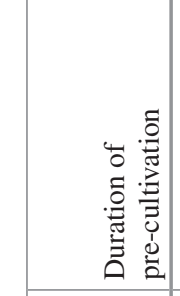 & & 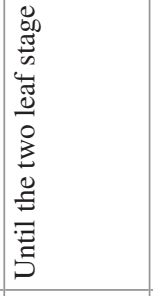 & 1 & $\bar{\lambda}$ & 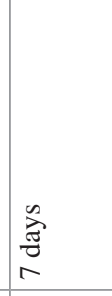 & 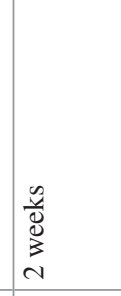 \\
\hline 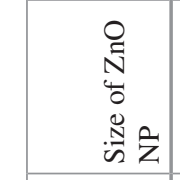 & 咅 & $\Xi$ & 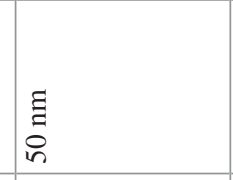 & 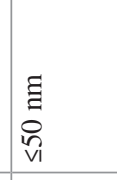 & 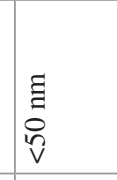 & $\exists$ \\
\hline 离 & 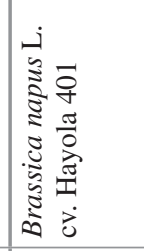 & 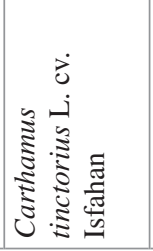 & 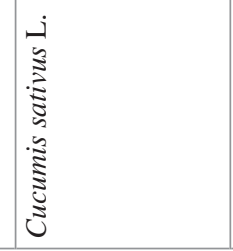 & 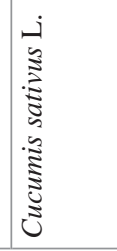 & 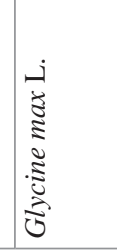 & 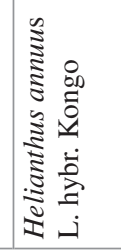 \\
\hline
\end{tabular}




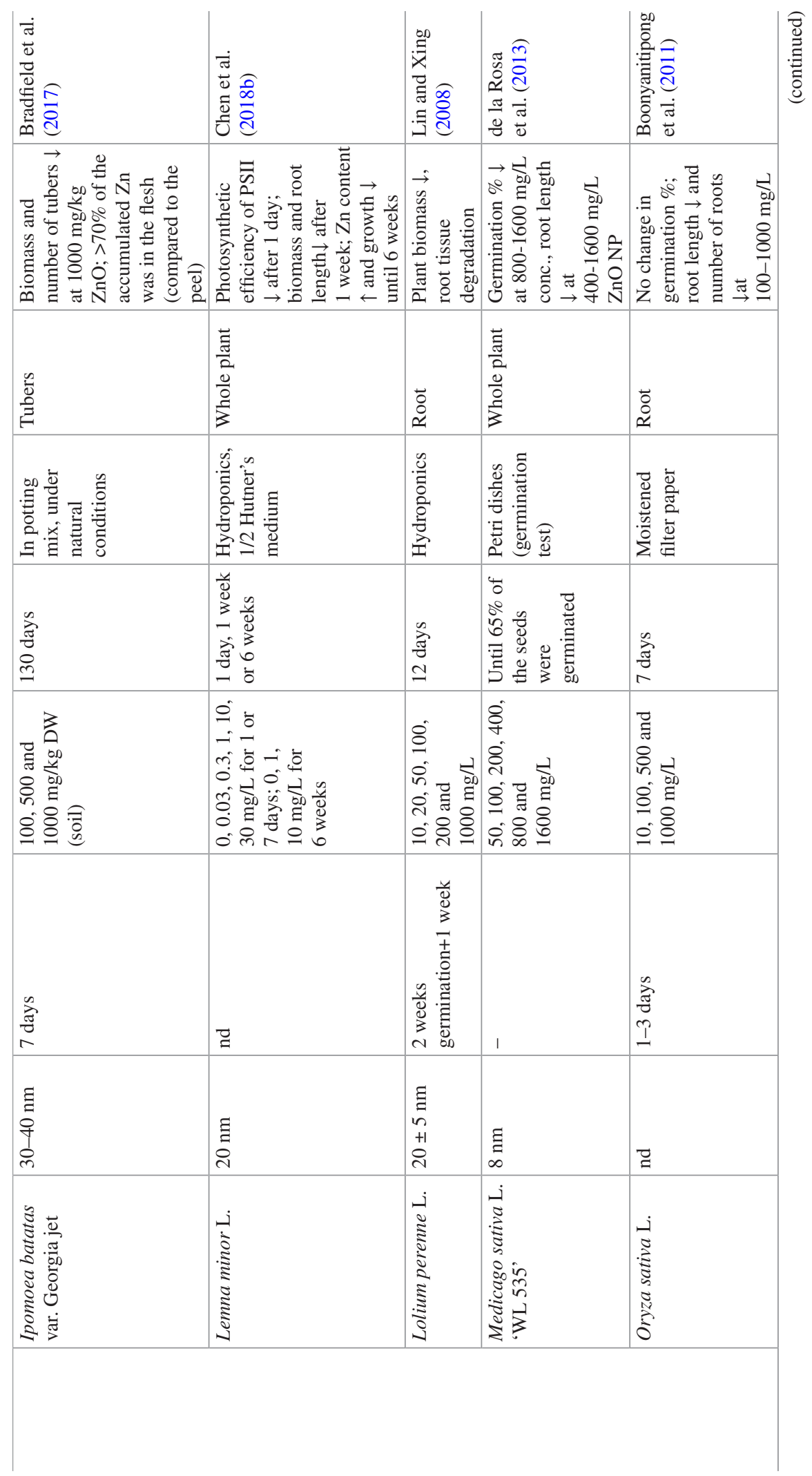




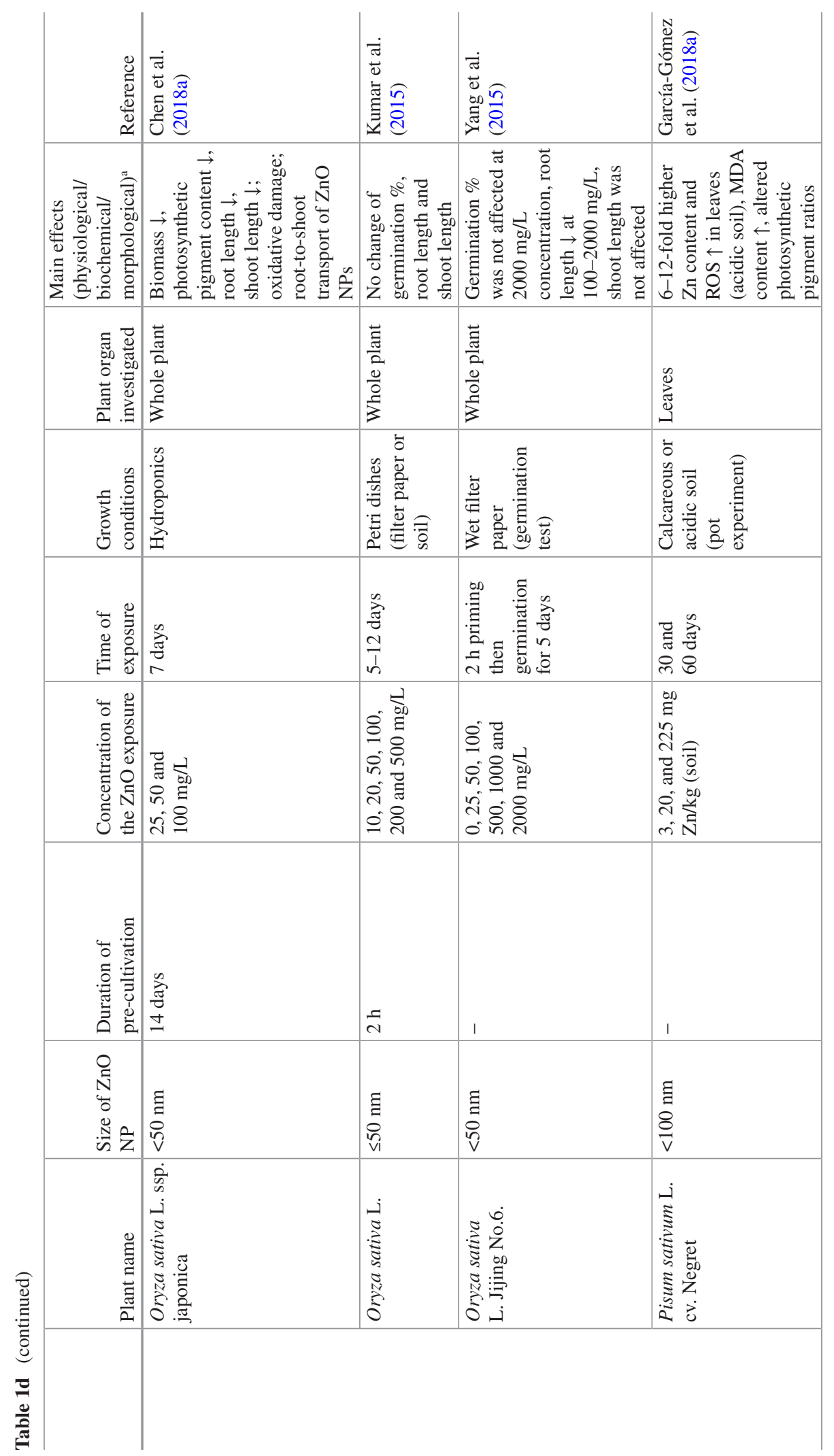




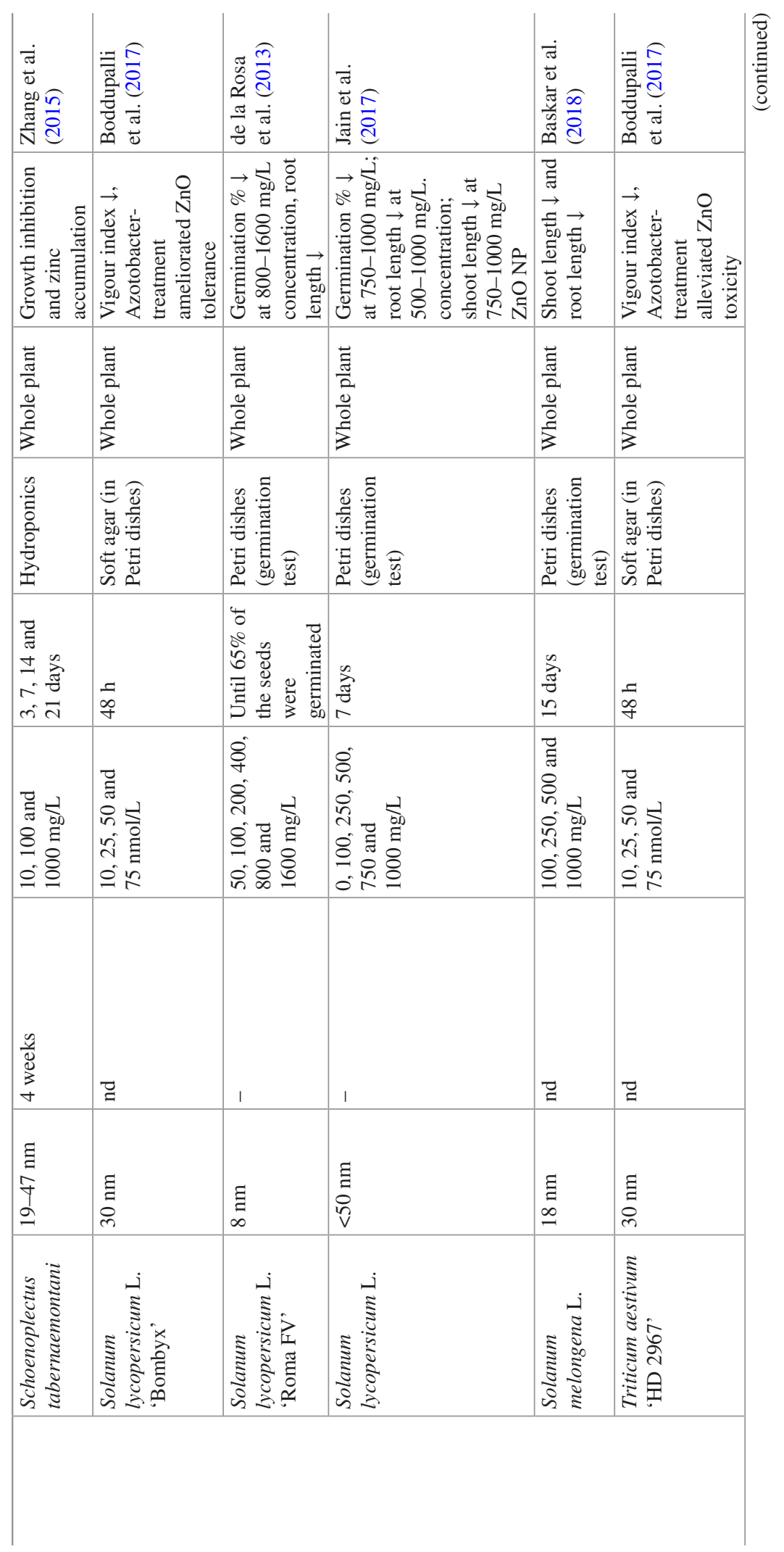




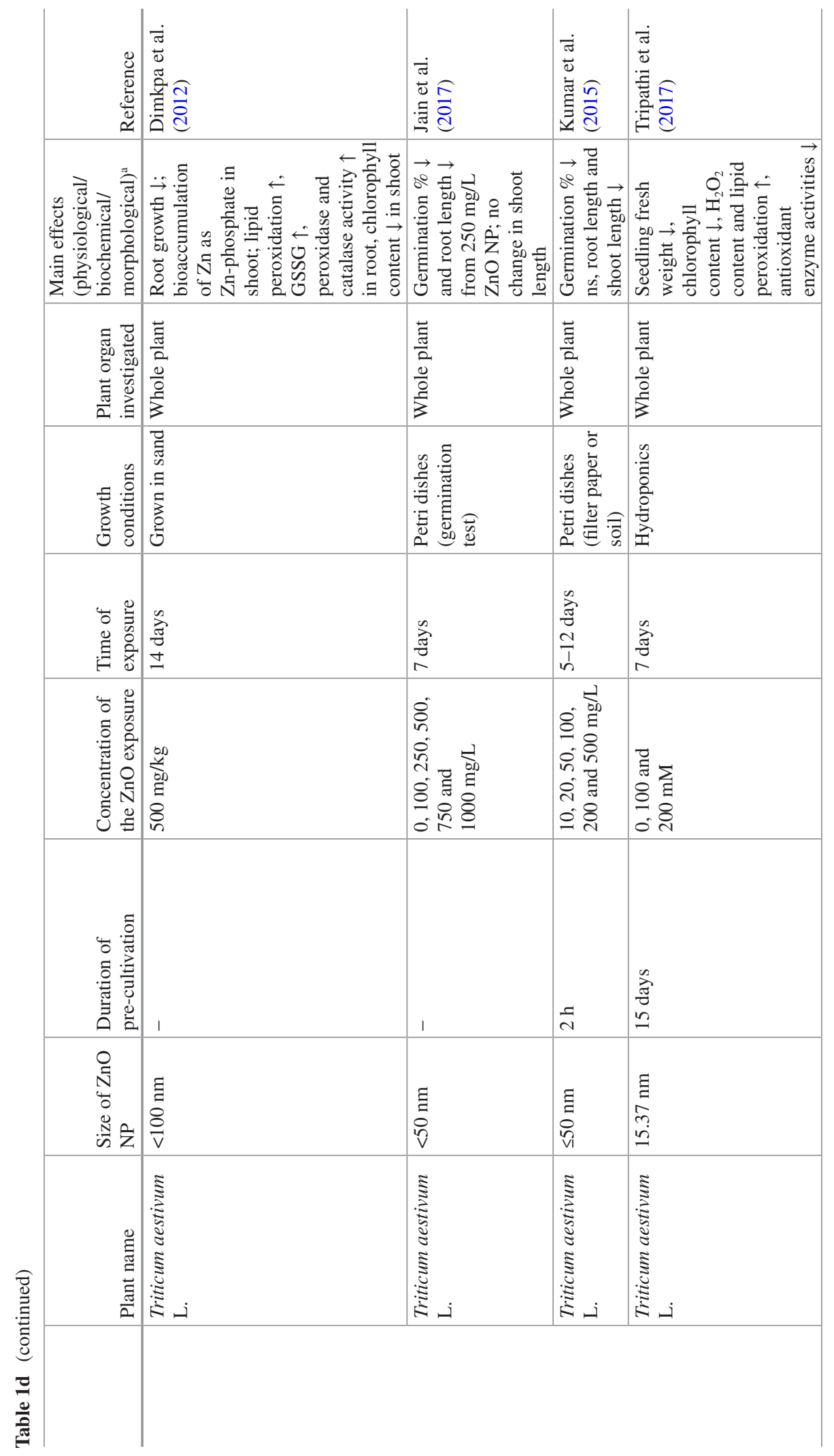




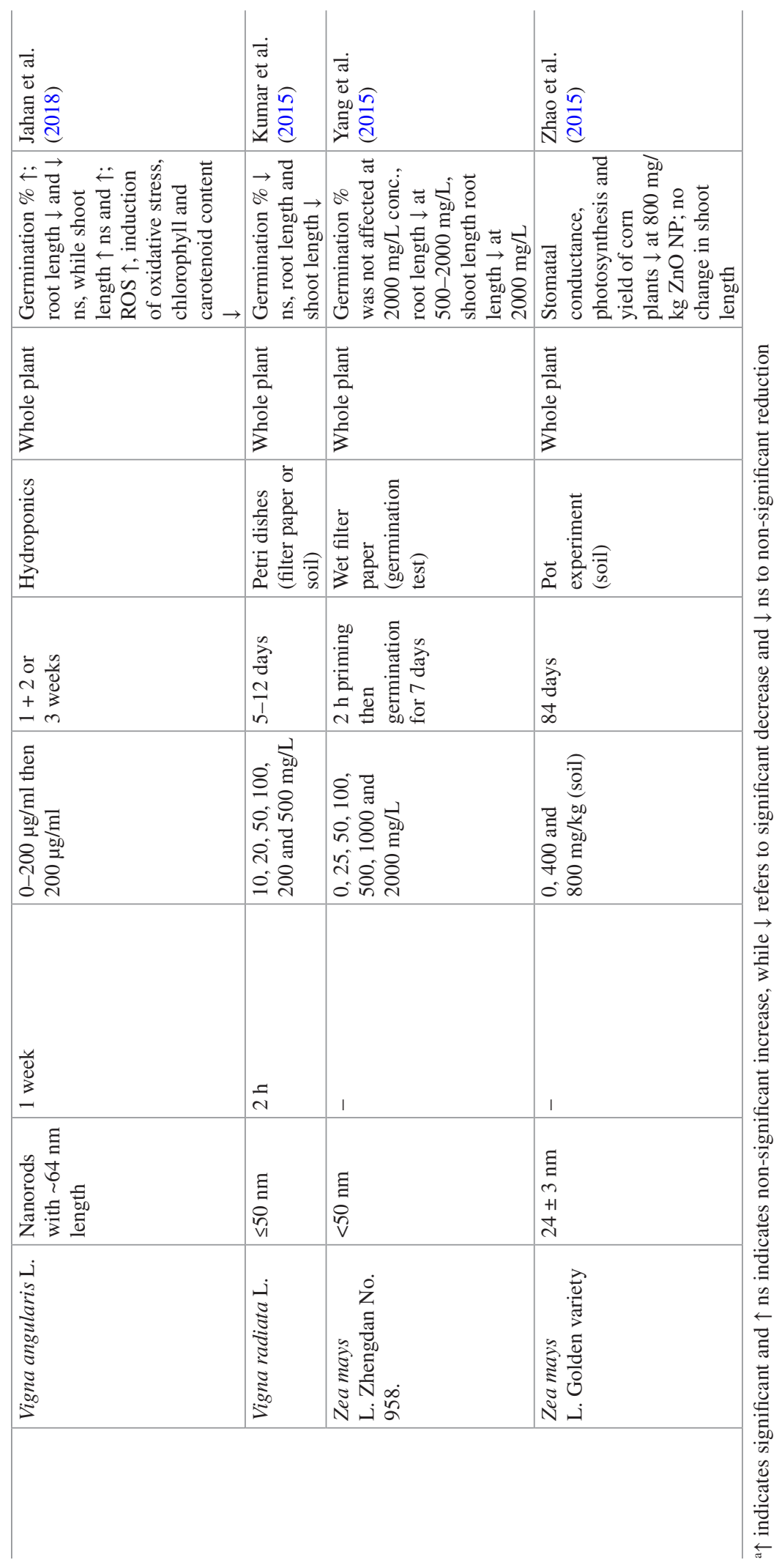


along with an antioxidant enzyme (SOD and POX) activity increase (Venkatachalam et al. 2017b; Table 1a).

Nonetheless, numerous studies focused on toxic effects, like oxidative stress and malondialdehyde (MDA) formation expressing lipid peroxidation as a response to larger doses of ZnO NPs. Mukherjee et al. (2014) described oxidative stress in green peas treated with $500 \mathrm{mg} / \mathrm{kg}$ (soil) ZnO NPs. An oxidative burst was observed in soybean (Hossain et al. 2016), in beet and pea (García-Gómez et al. 2018a) and in safflower (Hafizi and Nasr 2018) (Table 1d). In onion, a concentration-dependent increase of LP was detected, followed by a decreased mitotic index and an increased number of chromosomal aberrations suggesting a genotoxic effect of $\mathrm{ZnO}$ NPs (Kumari et al. 2011), which was further supported by Shaymurat et al. (2012) in garlic and Ghosh et al. (2016) in onion, tobacco and broad bean. Dose-dependent activation of SOD, CAT and ascorbate peroxidase (APX) was observed in tomato, while the plants showed growth retardation at higher (400-800 mg/L) ZnO NP concentration (Wang et al. 2018a; Table 1c). In Salicornia a significant increase in ROS and reactive nitrogen species (RNS) levels were displayed, coupled with a significant MDA increment. Peroxidase and APX activity declined, while Mn SOD, Fe SOD and cAPX were induced in response to the treatment (Balážová et al. 2018; Table 1c). Furthermore, in rice $\mathrm{ZnO}$ NP treatment triggered positive response of antioxidant enzymes was examined at molecular level, where levels of CSD1, CSD2, CATa, CATb, CATc, MSD1, FSD1, APXa and APXb were measured and mostly upregulated (Chen et al. 2018a). Summarily, we can say data published up to now suggest that $\mathrm{ZnO}$ NPs may act controversially in respect of oxidative processes depending on several factors like concentration, duration of exposure, age of the plant, the application of priming, etc.

\section{ZnO NPs Influence Nutrient Homeostasis and Photosynthetic Efficiency}

The last unexplained biochemical mechanism of $\mathrm{ZnO} \mathrm{NP}$ effect is the impact on nutrient homeostasis and photosynthesis. As seen previously, different concentrations of $\mathrm{ZnO}$ have different effects on photosynthesis ranging from beneficial to toxic effects. In cilantro (Pullagurala et al. 2018a) chlorophyll content increased in response to the treatment, the same as in case of peanut (Prasad et al. 2012), cotton (Venkatachalam et al. 2017b) or bean (Ewais et al. 2017) (Table 1a). Foliar application of $10 \mathrm{ppm} \mathrm{ZnO}$ caused an increment of phosphorus and chlorophyll content in cluster bean (Raliya and Tarafdar 2013). On the contrary, in green peas (Mukherjee et al. 2014), Indian mustard (Rao and Shekhawat 2014), corn (Zhao et al. 2015), Arabidopsis (Wang et al. 2015) and wheat (Tripathi et al. 2017) chlorophyll content attenuated in $\mathrm{ZnO}$-treated plants (Table 1d). In rice, a significant decline of chlorophyll content was observed and upon the examination of chlorophyll synthesis genes CHLD and CHLM expression levels reduced as response to the treatment 
cells, followed by the increment of root diameter (Balážová et al. 2018) or lateral root number (Nair and Chung 2017), which suggests the potential reorientation of root cells like in stress-induced morphogenic responses (SIMR, Potters et al. 2007) (Table 1c and 1d).

In the background of these negative processes, probably $\mathrm{Zn}$ content of the different plant organs was increased, causing changes in the physiological homeostasis, like lipid peroxidation, oxidative stress, nutrient imbalance or decreased protein production, as here we previously discussed.

\subsection{ZnO NP Affects Reproductive Processes}

Although there are many data about the impact of ZnO NPs on vegetative growth, it is noteworthy to mention that these agents may influence the reproductive traits of the plants, as well. There are both positive and negative impacts published. Laware and Raskar (2014) discovered that foliar spraying with ZnO NP may cause earlier flowering and elevated seed production of onion. Similarly, induced productivity of cowpea (Srinivasan et al. 2017; Table 1a) and bean (Ewais et al. 2017) was recorded after $\mathrm{ZnO} \mathrm{NP}$ foliar application. At the same time, in pot experiments filled with treated soil bean exhibited a decrease of fruit number and seed number per pod (Medina-Pérez et al. 2018).

\section{Stress Alleviation by $\mathrm{ZnO}$ NPs}

In some cases, stress-alleviating effect of $\mathrm{ZnO}$ NPs was also exhibited, for example in case of drought-stressed wheat (Taran et al. 2017), Cd- and Pb-stressed Leucaena leucocephala (Venkatachalam et al. 2017b) or As-treated rice (Wang et al. 2018b) (Table 1b).

\section{Conclusions and Future Perspectives}

Nowadays, $\mathrm{ZnO}$ nanoparticles (NPs) seem to be an indispensable part of our life due to the wide range of its usage (e.g. medicines with anticancer and antimicrobial activities or nanofertilisers in agriculture), therefore their emission to the environment and food chain remarkably has grown. Here, we tried to overview that plants being immovable how evolve strategies to protect themselves from these abiotic stress factors, but it was also proved that $\mathrm{ZnO}$ NPs may mitigate the negative effects of other toxic agents like heavy metals. Though there are an increasing number of reports dealing with the impact of $\mathrm{ZnO}$ NPs on plants, there is still little evidence of 
the potential translocation from root to shoot and there is only a few information about the anatomical changes in the root and/or shoot-like cell wall modifications triggered by $\mathrm{ZnO}$ NPs.

Acknowledgements This work was supported by the János Bolyai Research Scholarship of the Hungarian Academy of Sciences (Grant no. BO/00751/16/8) and by the National Research, Development and Innovation Fund (Grant no. NKFI-1 PD 131589, NKFI-6, K120383, NKFI KH 129511) and by UNKP-18-4 and UNKP-18-3-IV-SZTE-34 New National Excellence Program of the Ministry of Human Capacities.

\section{References}

Adams LK, Lyon DY, Alvarez PJ (2015) Comparative eco-toxicity of nanoscale $\mathrm{TiO}_{2}, \mathrm{SiO}_{2}$, and $\mathrm{ZnO}$ water suspensions. Water Nano-Micro Lett 7:219-242

Afrayeem SM, Chaurasia AK (2017) Effect of zinc oxide nanoparticles on seed germination and seed vigour in chilli (Capsicum аппиит L.). J Pharmacogn. Phytochemistry 6:1564-1566

Bacaksiz E, Parlak M, Tomakin M, Özcelik A, Karakiz M, Altunbas M (2008) The effect of zinc nitrate, zinc acetate and zinc chloride precursors on investigation of structural and optical properties of ZnO thin films. J Alloys Compd 466:447-450

Balážová L, Babula P, Baláž M, Bačkorová M, Bujňáková Z, Briančin J, Kurmanbayeva A, Sagi M (2018) Zinc oxide nanoparticles phytotoxicity on halophyte from genus Salicornia. Plant Physiol Biochem 130:30-42

Barhoumi L, Oukarroum A, Taher LB, Smiri LS, Abdelmelek H, Dewez D (2015) Effects of superparamagnetic iron oxide nanoparticles on photosynthesis and growth of the aquatic plant Lemna gibba. Arch Environ Contam Toxicol 68:510-520

Baskar V, Nayeem S, Kuppuraj SP, Muthu T, Ramalingam S (2018) Assessment of the effects of metal oxide nanoparticles on the growth, physiology and metabolic responses in in vitro grown eggplant (Solanum melongena). 3 Biotech 8:362

Bell PF, McLaughlin MJ, Cozens G, Stevens DP, Owens G, South H (2003) Plant uptake of 14C-citrate, and 14C-histidine from chelator-buffered and conventional hydroponic solutions. Plant Soil 253:311-319

Bhattacharyya S, Gedanken A (2007) A template-free, sonochemical route to porous $\mathrm{ZnO}$ nanodisks. Microporous Mesoporous Mater 110:553-559

Boddupalli A, Tiwari R, Sharma A, Singh S, Prasanna R, Nain L (2017) Elucidating the interactions and phytotoxicity of zinc oxide nanoparticles with agriculturally beneficial bacteria and selected crop plants. Folia Microbiol 62:253-262

Boonyanitipong P, Kositsup B, Kumar P, Baruah S, Dutta J (2011) Toxicity of ZnO and $\mathrm{TiO}_{2}$ nanoparticles on germinating rice seed Oryza sativa $\mathrm{L}$. Int $\mathrm{J}$ Biosci Biochem Bioinform $1: 282-285$

Bradfield SJ, Kumar P, White JC, Ebbs SD (2017) Zinc, copper, or cerium accumulation from metal oxide nanoparticles or ions in sweet potato: yield effects and projected dietary intake from consumption. Plant Physiol Biochem 110:128-137

Brayner R, Ferrari-lliou R, Brivois N, Djediat S, Benedetti MF, Fiévet F (2006) Toxicological impact studies based on Escherichia coli bacteria in ultrafine $\mathrm{ZnO}$ nanoparticles colloidal medium. Nano Lett 6:866-870

Brunner TJ, Wick P, Manser P, Spohn P, Grass RN, Limbach LK, Bruinink A, Stark WJ (2006) In vitro cytotoxicity of oxide nanoparticles: comparison to asbestos, silica, and the effect of particle solubility. Environ Sci Technol 40:4374-4381

Burman U, Saini M, Kumar P (2013) Effect of zinc oxide nanoparticles on growth and antioxidant system of chickpea seedlings. Toxicol Environ Chem 95:605-612 
Carpita N, Sabularse D, Montezinos D, Delmer DP (1979) Determination of the pore size of cell walls of living plant cells. Science 205:1144-1147

Chaari M, Matoussi A (2012) Electrical conduction and dielectric studies of $\mathrm{ZnO}$ pellets. Phys B Condens Matter 407:3441-3447

Chen J, Dou R, Yang Z, You T, Gao X, Wang L (2018a) Phytotoxicity and bioaccumulation of zinc oxide nanoparticles in rice (Oryza sativa L.). Plant Physiol Biochem 130:604-612

Chen X, O'Halloran J, Jansen MA (2018b) Time matters: the toxicity of zinc oxide nanoparticles to Lemna minor L. increases with exposure time. Water Air Soil Pollut 229:99

Clemens S (2001) Molecular mechanisms of plant metal tolerance and homeostasis. Planta 212:475-486

Darlington TK, Neigh AM, Spencer MT, Nguyen OT, Oldenburg SJ (2009) Nanoparticle characteristics affecting environmental fate and transport through soil. Environ Toxicol Chem 28:1101-1199

de la Rosa G, López-Moreno ML, de Haro D, Botez CE, Peralta-Videa JR, Gardea-Torresdey JL (2013) Effects of $\mathrm{ZnO}$ nanoparticles in alfalfa, tomato, and cucumber at the germination stage: root development and X-ray absorption spectroscopy studies. Pure Appl Chem 85:2161-2174

Demir E, Kaya N, Kaya B (2014) Genotoxic effects of zinc oxide and titanium dioxide nanoparticles on root meristem cells of Allium cepa by comet assay. Turk J Biol 38:31-39

Dewez D, Oukarroum A (2012) Silver nanoparticles toxicity effect on photosystem II photochemistry of the green alga Chlamydomonas reinhardtii treated in light and dark conditions. Toxicol Environ Chem 94:1536-1546

Dimkpa CO, McLean JE, Latta DE, Manangón E, Britt DW, Johnson WP, Boyanov MI, Anderson $\mathrm{AJ}$ (2012) $\mathrm{CuO}$ and $\mathrm{ZnO}$ nanoparticles: phytotoxicity, metal speciation, and induction of oxidative stress in sand-grown wheat. J Nanopart Res 14:1125

Doğaroğlu ZG, Köleli N (2017) $\mathrm{TiO} 2$ and $\mathrm{ZnO}$ nanoparticles toxicity in barley (Hordeum vulgare L.). Clean 45:1700096

Ebbs S, Uchil S (2008) Cadmium and zinc induced chlorosis in Indian mustard (Brassica juncea (L.) Czern) involves preferential loss of chlorophyll b. Photosynthetica 46:49-55

Ebbs SD, Bradfield SJ, Kumar P, White JC, Musante C, Ma X (2016) Accumulation of zinc, copper, or cerium in carrot (Daucus carota) exposed to metal oxide nanoparticles and metal ions. Environ Sci Nano 3:114-126

Elizabath A, Bahadur V, Misra P, Prasad VM, Thomas T (2017) Effect of different concentrations of iron oxide and zinc oxide nanoparticles on growth and yield of carrot (Daucus carota L.). J Pharmacogn Phytochem 6:1266-1269

Elmer WH, Ma C, White JC (2018) Nanoparticles for plant disease management. Curr Opin Environ Sci Health 6:66-70

Ewais EA, Ismail MA, Badawy AA (2017) Vegetative growth, photosynthetic pigments and yield of Phaseolus vulgaris (L.) plants in response to the application of biologically-synthesized zinc oxide nanoparticles and zinc sulfate. Al Azhar Bulletin of Science Vol. 9th., Conf., March 2017, pp 33-46

Faizan M, Faraz A, Yusuf M, Khan ST, Hayat S (2018) Zinc oxide nanoparticle-mediated changes in photosynthetic efficiency and antioxidant system of tomato plants. Photosynthetica 56:678-686

García-Gómez C, García S, Obrador AF, González D, Babín M, Fernández MD (2018a) Effects of aged $\mathrm{ZnO}$ NPs and soil type on $\mathrm{Zn}$ availability, accumulation and toxicity to pea and beet in a greenhouse experiment. Ecotoxicol Environ Saf 160:222-230

García-Gómez C, Obrador A, González D, Babín M, Fernández MD (2018b) Comparative study of the phytotoxicity of $\mathrm{ZnO}$ nanoparticles and $\mathrm{Zn}$ accumulation in nine crops grown in a calcareous soil and an acidic soil. Sci Total Environ 644:770-780

Ghodake G, Seo YD, Lee DS (2011) Hazardous phytotoxic nature of cobalt and zinc oxide nanoparticles assessed using Allium сера. J Hazard Mater 186:952-955 
Ghosh M, Jana A, Sinha S, Jothiramajayam M, Nag A, Chakraborty A, Mukherjee A, Mukherjee A (2016) Effects of $\mathrm{ZnO}$ nanoparticles in plants: cytotoxicity, genotoxicity, deregulation of antioxidant defenses, and cell-cycle arrest. Mutat Res Genet Toxicol Environ Mutagen 807:25-32

Hafizi Z, Nasr N (2018) The effect of zinc oxide nanoparticles on safflower plant growth and physiology. Eng Technol Appl Sci Res 8:2508-2513

Hernandez-Viezcas JA, Castillo-Michel H, Servin AD, Peralta-Videa JR, Gardea-Torresdey JL (2011) Spectroscopic verification of zinc absorption and distribution in the desert plant Prosopis juliflora-velutina (velvet mesquite) grown with $\mathrm{ZnO}$ nanoparticles. Chem Eng J 170:346-352

Hossain Z, Mustafa G, Sakata K, Komatsu S (2016) Insights into the proteomic response of soybean towards Al2O3, ZnO, and Ag nanoparticles stress. J Hazard Mater 304:291-305

Hou J, Wu Y, Li X, Wei B, Li S, Wang X (2018) Toxic effects of different types of zinc oxide nanoparticles on algae, plants, invertebrates, vertebrates and microorganisms. Chemosphere 193:852-860

Hu C, Liu X, Li X, Zhao Y (2014) Evaluation of growth and biochemical indicators of Salvinia natans exposed to zinc oxide nanoparticles and zinc accumulation in plants. Environ Sci Pollut Res 21:732-739

Huang Z, Zheng X, Yan D, Yin G, Liao X, Kang Y, Yao Y, Huang D, Hao B (2008) Toxicological effect of ZnO nanoparticles based on bacteria. Langmuir 24:4140-4144

Hussain A, Ali S, Rizwan M, ur Rehman MZ, Javed MR, Imran M, Chatha SAS, Nazir R (2018) Zinc oxide nanoparticles alter the wheat physiological response and reduce the cadmium uptake by plants. Environ Pollut 242:1518-1526

Jahan S, Alias YB, Bakar AFBA, Yusoff IB (2018) Toxicity evaluation of $\mathrm{ZnO}$ and $\mathrm{TiO}_{2}$ nanomaterials in hydroponic red bean (Vigna angularis) plant: physiology, biochemistry and kinetic transport. J Environ Sci 72:140-152

Jain A, Sinilal B, Dhandapani G, Meagher RB, Sahi SV (2013) Effects of deficiency and excess of zinc on morpho-physiological traits and spatiotemporal regulation of zinc responsive genes reveal incidence of cross talk between micro and macronutrients. Environ Sci Technol 47:5327-5335

Jain N, Bhargava A, Pareek V, Akhtar MS, Panwar J (2017) Does seed size and surface anatomy play role in combating phytotoxicity of nanoparticles? Ecotoxicology 26:238-249

Jalal R, Goharshadi EK, Abareshi M, Moosavi M, Yousefi A, Nancarrow P (2010) ZnO nanofluids: green synthesis, characterization, and antibacterial activity. Mater Chem Phys 121:198-201

Javed R, Usma M, Yücesan B, Zia M, Gürel E (2017) Effect of zinc oxide (ZnO) nanoparticles on physiology and steviol glycosides production in micropropagated shoots of Stevia rebaudiana Bertoni. Plant Physiol Biochem 110:94-99

Jayarambabu N, Kumari BS, Rao KV, Prabhu YT (2014) Germination and growth characteristics of mungbean seeds (Vigna radiata $\mathrm{L}$.) affected by synthesized zinc oxide nanoparticles. Int $\mathrm{J}$ Curr Eng Technol 4:2347-5161

Jiang HM, Yang JC, Zhang JF (2007) Effects of external phosphorus on the cell ultrastructure and the chlorophyll content of maize under cadmium and zinc stress. Environ Pollut 147:750-756

Kim S, Kim J, Lee I (2011) Effects of $\mathrm{Zn}$ and $\mathrm{ZnO}$ nanoparticles and $\mathrm{Zn}^{2+}$ on soil enzyme activity and bioaccumulation of $\mathrm{Zn}$ in Cucumis sativus. Chem Ecol 27:49-55

Kołodziejczak-Radzimska A, Jesionowski T (2014) Zinc oxide-from synthesis to application: a review. Materials 7:2833-2881

Kouhi SMM, Lahouti M, Ganjeali A, Entezari MH (2014) Comparative phytotoxicity of ZnO nanoparticles, $\mathrm{ZnO}$ microparticles, and $\mathrm{Zn}^{2+}$ on rapeseed (Brassica napus L.): investigating a wide range of concentrations. Toxicol Environ Chem 96:861-868

Kumar S, Patra AK, Datta SC, Rosin KG, Purakayastha TJ (2015) Phytotoxicity of nanoparticles to seed germination of plants. Int J Adv Res 3:854-865

Kumar UJ, Bahadur V, Prasad VM, Mishra S, Shukla PK (2017) Effect of different concentrations of iron oxide and zinc oxide nanoparticles on growth and yield of strawberry (Fragaria x ananassa Duch) cv. Chandler. Int J Curr Microbiol App Sci 6:2440-2445 
Kumari M, Khan SS, Pakrashi S, Mukherjee A, Chandrasekaran N (2011) Cytogenetic and genotoxic effects of zinc oxide nanoparticles on root cells of Allium cepa. J Hazard Mater 190:613-621

Laware SL, Raskar S (2014) Influence of zinc oxide nanoparticles on growth, flowering and seed productivity in onion. Int J Curr Microbiol App Sci 3:874-881

Lee CW, Mahendra S, Zodrow K, Li D, Tsai YC, Braam J, Alvarez PJ (2010) Developmental phytotoxicity of metal oxide nanoparticles to Arabidopsis thaliana. Environ Toxicol Chem 29:669-675

Lee S, Chung H, Kim S, Lee I (2013) The genotoxic effect of $\mathrm{ZnO}$ and $\mathrm{CuO}$ nanoparticles on early growth of buckwheat, Fagopyrum esculentum. Water Air Soil Pollut 224:1668

Lin D, Xing B (2008) Root uptake and phytotoxicity of $\mathrm{ZnO}$ nanoparticles. Environ Sci Technol 42:5580-5585

Lipovsky A, Tzitrinovich Z, Friedmann H, Applerot G, Gedanken A, Lubart R (2009) EPR study of visible light-induced ROS generation by nanoparticles of $\mathrm{ZnO}$. J Phys Chem C 113:15997-16001

López-Moreno ML, de la Rosa G, Hernández-Viezcas JÁ, Castillo-Michel H, Botez CE, PeraltaVidea JR, Gardea-Torresdey JL (2010) Evidence of the differential biotransformation and genotoxicity of $\mathrm{ZnO}$ and $\mathrm{CeO}_{2}$ nanoparticles on soybean (Glycine max) plants. Environ Sci Technol 44:7315-7320

López-Moreno ML, de la Rosa G, Cruz-Jiménez G, Castellano L, Peralta-Videa JR, GardeaTorresdey JL (2017) Effect of $\mathrm{ZnO}$ nanoparticles on corn seedlings at different temperatures; X-ray absorption spectroscopy and ICP/OES studies. Microchem J 134:54-61

Lou X (1991) Development of ZnO series ceramic semiconductor gas sensors. J Sens Trans Technol 3:1-5

Ludi B, Niederberger M (2013) Zinc oxide nanoparticles: chemical mechanism and classical and non-classical crystallization. Dalton Trans 42:12554-12568

Lv J, Zhang S, Luo L, Zhang J, Yang K, Christie P (2015) Accumulation, speciation and uptake pathway of $\mathrm{ZnO}$ nanoparticles in maize. Environ Sci Nano 2:68-77

Maity A, Natarajan N, Vijay D, Srinivasan R, Pastor M, Malaviya DR (2018) Influence of metal nanoparticles (NPs) on germination and yield of oat (Avena sativa) and berseem (Trifolium alexandrinum). Proc Natl Acad Sci India Sect B Biol Sci 88:595-607

Marslin G, Sheeba CJ, Franklin G (2017) Nanoparticles alter secondary metabolism in plants via ROS burst. Front Plant Sci 8:832

Medina-Pérez G, Fernandez-Luqueno F, TREJO-TÉLLEZ LI, Lopez-Valdez F, PampillonGonzalez L (2018) Growth and development of common bean (Phaseolus vulgaris 1.) var. pinto saltillo exposed to iron, titanium, and zinc oxide nanoparticles in an agricultural soil. Appl Ecol Environ Res 16:1883-1897

Medina-Velo IA, Barrios AC, Zuverza-Mena N, Hernandez-Viezcas JA, Chang CH, Ji Z, Zink JI, Peralta-Videa JR, Gardea-Torresdey JL (2017) Comparison of the effects of commercial coated and uncoated $\mathrm{ZnO}$ nanomaterials and $\mathrm{Zn}$ compounds in kidney bean (Phaseolus vulgaris) plants. J Hazard Mater 332:214-222

Medina-Velo IA, Zuverza-Mena N, Tamez C, Ye Y, Hernandez-Viezcas JA, White JC, PeraltaVidea JR, Gardea-Torresdey JL (2018) Minimal transgenerational effect of ZnO nanomaterials on the physiology and nutrient profile of Phaseolus vulgaris. ACS Sustain Chem Eng 6:7924-7930

Méndez-Argüello B, Vera-Reyes I, Mendoza-Mendoza E, García-Cerda LA, Puente-Urbina BA, Saldívar RHL (2016) Growth promotion of Capsicum annuum plants by zinc oxide nanoparticles. Nova Sci 8:140-156

Mishra PK, Mishra H, Ekielski A, Talegaonkar S, Vaidya B (2017) Zinc oxide nanoparticles: a promising nanomaterial for biomedical applications. Drug Discov Today 22:1825-1834

Moezzi A, McDonagh AM, Cortie MB (2012) Zinc oxide particles: synthesis, properties and applications. Chem Eng J 185:1-22 
Mukherjee A, Peralta-Videa JR, Bandyopadhyay S, Rico CM, Zhao L, Gardea-Torresdey JL (2014) Physiological effects of nanoparticulate $\mathrm{ZnO}$ in green peas (Pisum sativum L.) cultivated in soil. Metallomics 6:132-138

Mukherjee A, Sun Y, Morelius E, Tamez C, Bandyopadhyay S, Niu G, White JC, Peralta-Videa JR, Gardea-Torresdey JL (2016) Differential toxicity of bare and hybrid ZnO nanoparticles in green pea (Pisum sativum L.): a life cycle study. Front Plant Sci 6:1242

Munir T, Rizwan M, Kashif M, Shahzad A, Ali S, Amin N, Zahid R, Alam MFE, Imran M (2018) Effect of zinc oxide nanoparticles on the growth and Zn uptake in wheat (Triticum aestivum L.) by seed priming method. Dig J Nanomater Biostruct 13:315-323

Nair PMG, Chung IM (2017) Regulation of morphological, molecular and nutrient status in Arabidopsis thaliana seedlings in response to $\mathrm{ZnO}$ nanoparticles and $\mathrm{Zn}$ ion exposure. Sci Total Environ 575:187-198

Narendhran S, Rajiv P, Sivaraj R (2016) Influence of zinc oxide nanoparticles on growth of Sesamum indicum L. in zinc deficient soil. Int J Pharm Pharm Sci 8:365-371

Özgür Ü, Alivov YI, Liu C, Teke A, Reshchikov MA, Doğan S, Avrutin V, Cho SJ, Morkoç H (2005) A comprehensive review of ZnO materials and devices. J Appl Phys 98:041301. https:// doi.org/10.1063/1.1992666

Padmavathy N, Vijayaraghavan R (2008) Enhanced bioactivity of ZnO nanoparticles-an antimicrobial study. Sci Technol Adv Mater 9:035004. https://doi.org/10.1088/1468-6996/9/3/035004

Pence NS, Larsen PB, Ebbs SD, Letham DLD, Lasat MM, Garvin DF, Eide D, Kochian V (2000) The molecular physiology of heavy metal transport in the $\mathrm{Zn} / \mathrm{Cd}$ hyperaccumulator Thlaspi caerulescens. Proc Natl Acad Sci U S A 97:4956-4960

Peralta-Videa JR, Hernandez-Viezcas JA, Zhao L, Diaz BC, Ge Y, Priester JH, Holden PA, GardeaTorresdey JL (2014) Cerium dioxide and zinc oxide nanoparticles alter the nutritional value of soil cultivated soybean plants. Plant Physiol Biochem 80:128-135

Pokhrel LR, Dubey B (2013) Evaluation of developmental responses of two crop plants exposed to silver and zinc oxide nanoparticles. Sci Total Environ 452:321-332

Potters G, Pasternak TP, Guisez Y, Palme KJ, Jansen MA (2007) Stress-induced morphogenic responses: growing out of trouble? Trends Plant Sci 12:98-105

Poynton HC, Lazorchak JM, Impellitteri CA, Smith ME, Rogers K, Patra M, Hammer KA, Allen HJ, Vulpe CD (2011) Differential gene expression in Daphnia magna suggests distinct modes of action and bioavailability for $\mathrm{ZnO}$ nanoparticles and $\mathrm{Zn}$ ions. Environ Sci Technol 45:762-768

Prasad TNVKV, Sudhakar P, Sreenivasulu Y, Latha P, Munaswamy V, Reddy KR, Sreeprasad TS, Sajanlal PR, Pradeep T (2012) Effect of nanoscale zinc oxide particles on the germination, growth and yield of peanut. J Plant Nutr 35:905-927

Pullagurala VLR, Adisa IO, Rawat S, Kalagara S, Hernandez-Viezcas JA, Peralta-Videa JR, Gardea-Torresdey JL (2018a) ZnO nanoparticles increase photosynthetic pigments and decrease lipid peroxidation in soil grown cilantro (Coriandrum sativum). Plant Physiol Biochem 132:120-127

Pullagurala VLR, Adisa IO, Rawat S, Kim B, Barrios AC, Medina-Velo IA, Hernandez-Viezcas JA, Peralta-Videa JR, Gardea-Torresdey JL (2018b) Finding the conditions for the beneficial use of $\mathrm{ZnO}$ nanoparticles towards plants-a review. Environ Pollut 241:1175-1181

Raliya R, Tarafdar JC (2013) ZnO nanoparticle biosynthesis and its effect on phosphorousmobilizing enzyme secretion and gum contents in cluster bean (Cyamopsis tetragonoloba L.). Agric Res 2:48-57

Raliya R, Nair R, Chavalmane S, Wang WN, Biswas P (2015) Mechanistic evaluation of translocation and physiological impact of titanium dioxide and zinc oxide nanoparticles on the tomato (Solanum lycopersicum L.) plant. Metallomics 7:1584-1594

Rao S, Shekhawat GS (2014) Toxicity of ZnO engineered nanoparticles and evaluation of their effect on growth, metabolism and tissue specific accumulation in Brassica juncea. J Environ Chem Eng 2:105-114 
Raskar SV, Laware SL (2014) Effect of zinc oxide nanoparticles on cytology and seed germination in onion. Int J Curr Microbiol App Sci 3:467-473

Rizwan M, Ali S, Ali B, Adrees M, Arshad M, Hussain A, Rehman MZ, Waris AA (2019) Zinc and iron oxide nanoparticles improved the plant growth and reduced the oxidative stress and cadmium concentration in wheat. Chemosphere 214:269-277

Sawai J, Shoji S, Igarashi I, Hashimoto A, Kokugan T, Shimizu M, Kojima H (1998) Hydrogen peroxide as an antibacterial factor in zinc oxide powder slurry. J Ferment Bioeng 86:521-522

Scheckel KG, Luxton TP, Badawy AME, Impellitteri CA, Tolaymat TM (2010) Synchrotron speciation of silver and zinc oxide nanoparticles aged in a kaolin suspension. Environ Sci Technol 44:1307-1312

Segets D, Gradl J, Taylor RK, Vassilev V, Peukert W (2009) Analysis of optical absorbance spectra for the determination of $\mathrm{ZnO}$ nanoparticle size distribution, solubility, and surface energy. ACS Nano 3:1703-1710

Seven O, Dindar B, Aydemir S, Metin D, Ozinel O, Icli S (2004) Solar photocatalytic disinfection of a group of bacteria and fungi aqueous suspensions with $\mathrm{TiO}_{2}, \mathrm{ZnO}$ and Sahara Desert dust. J Photochem Photobiol A 165:103-107

Shaymurat T, Gu J, Xu C, Yang Z, Zhao Q, Liu Y, Liu Y (2012) Phytotoxic and genotoxic effects of $\mathrm{ZnO}$ nanoparticles on garlic (Allium sativum L.): a morphological study. Nanotoxicology 6:241-248

Siddiqui ZA, Khan MR, Abd-Allah EF, Parveen A (2018) Titanium dioxide and zinc oxide nanoparticles affect some bacterial diseases, and growth and physiological changes of beetroot. Int J Veg Sci 25:409. https://doi.org/10.1080/19315260.2018.1523267

Sirelkhatim A, Mahmud S, Seeni A, Kaus NHM, Ann LC, Bakhori SKM, Hasan H, Mohamad D (2015) Review on zinc oxide nanoparticles: antibacterial activity and toxicity mechanism. Nano-Micro Lett 7:219-242

Souza JF, Dolder H, Cortelazzo AL (2005) Effect of excess cadmium and zinc ions on roots and shoots of maize seedlings. J Plant Nutr 28:1923-1931

Srinivasan R, Maity A, Singh KK, Ghosh PK, Kumar S, Srivastava MK, Radhakrishna A, Srivastava R, Kumari B (2017) Influence of copper oxide and zinc oxide nano-particles on growth of fodder cowpea and soil microbiological properties. Range Manag Agrofor 38:208-214

Stanković A, Dimitrijević S, Uskoković D (2013) Influence of size scale and morphology on antibacterial properties of $\mathrm{ZnO}$ powders hydrothermally synthesized using different surface stabilizing agents. Colloids Surf B Biointerfaces 102:21-28

Stoimenov PK, Klinger RL, Marchin GL, Klabunde KJ (2002) Metal oxide nanoparticles as bactericidal agents. Langmuir 18:6679-6686

Sturikova H, Krystofova O, Hedbravny J, Vojtech A (2018) The comparison of effect of zinc sulfhate and zinc oxide nanoparticles on plants. In: Mendel Net Conference Brono, November 8-9, 2017, pp 932-936

Suriyaprabha R, Sreeja KA, Prabu M, Prabu P, Rajendran V (2018) Bioaccumulation of transition metal oxide nanoparticles and their influence on early growth stages of Vigna unguiculata seeds. BioNanoScience 8:752-760

Taran N, Storozhenko V, Svietlova N, Batsmanova L, Shvartau V, Kovalenko M (2017) Effect of zinc and copper nanoparticles on drought resistance of wheat seedlings. Nanoscale Res Lett 12:60

The Global Market for Metal and Metal Oxide Nanoparticles 2010-2027; ID: 4318916; http://www. researchandmarkets.com/reports/2488811/the_global_market_for_metal_oxide_nanoparticles

Tilney LG, Cooke TJ, Connelly PS, Tilney MS (1991) The structure of plasmodesmata as revealed by plasmolysis, detergent extraction, and protease digestion. J Cell Biol 112:739-747

Tripathi DK, Mishra RK, Singh S, Singh S, Singh VP, Singh PK, Chauhan DK, Prasad SM, Dubey NK, Pandey AC (2017) Nitric oxide ameliorates zinc oxide nanoparticles phytotoxicity in wheat seedlings: implication of the ascorbate-glutathione cycle. Front Plant Sci 8:1 
Venkatachalam P, Jayaraj M, Manikandan R, Geetha N, Rene ER, Sharma NC, Sahi SV (2017a) Zinc oxide nanoparticles (ZnONPs) alleviate heavy metal-induced toxicity in Leucaena leucocephala seedlings: a physiochemical analysis. Plant Physiol Biochem 110:59-69

Venkatachalam P, Priyanka N, Manikandan K, Ganeshbabu I, Indiraarulselvi P, Geetha N, Muralikrishna K, Bhattacharya RC, Tiwari M, Sharma N, Sahi SV (2017b) Enhanced plant growth promoting role of phycomolecules coated zinc oxide nanoparticles with $\mathrm{P}$ supplementation in cotton (Gossypium hirsutum L.). Plant Physiol Biochem 110:118-127

Wang ZL (2008) Splendid one-dimensional nanostructures of zinc oxide: a new nanomaterial family for nanotechnology. ACS Nano 2:1987-1992

Wang J, Cao J, Fang B, Lu P, Deng S, Wang H (2005) Synthesis and characterization of multipod, flower-like, and shuttle-like $\mathrm{ZnO}$ frameworks in ionic liquids. Mater Lett 59:1405-1408

Wang C, Liu H, Chen J, Tian Y, Shi J, Li D, Guo C, Ma Q (2014) Carboxylated multi-walled carbon nanotubes aggravated biochemical and subcellular damages in leaves of broad bean (Vicia $f a b a$ L.) seedlings under combined stress of lead and cadmium. J Hazard Mater 274:404-412

Wang X, Yang X, Chen S, Li Q, Wang QW, Hou C, Gao X, Li W, Shucai W (2015) Zinc oxide nanoparticles affect biomass accumulation and photosynthesis in Arabidopsis. Front Plant Sci 6:1243

Wang XP, Li QQ, Pei ZM, Wang SC (2018a) Effects of zinc oxide nanoparticles on the growth, photosynthetic traits, and antioxidative enzymes in tomato plants. Biol Plant 62:801-808

Wang X, Sun W, Zhang S, Sharifan H, Ma X (2018b) Elucidating the effects of cerium oxide nanoparticles and zinc oxide nanoparticles on arsenic uptake and speciation in rice (Oryza sativa) in a hydroponic system. Environ Sci Technol 52:10040-10047

Watson J, Fang T, Dimkpa CO, Britt DW, McLean JE, Jacobson A, Anderson AJ (2015) The phytotoxicity of $\mathrm{ZnO}$ nanoparticles on wheat varies with soil properties. Biometals 28:101-112

Xia T, Kovochich M, Liong M, Mädler L, Gilbert B, Shi H, Yeh JI, Zink JI, Nel AE (2008) Comparison of the mechanism of toxicity of zinc oxide and cerium oxide nanoparticles based on dissolution and oxidative stress properties. ACS Nano 2:2121-2134

Xu J, Luo X, Wang Y, Feng Y (2018) Evaluation of zinc oxide nanoparticles on lettuce (Lactuca sativa L.) growth and soil bacterial community. Environ Sci Pollut Res 25:6026-6035

Yang Z, Chen J, Dou R, Gao X, Mao C, Wang L (2015) Assessment of the phytotoxicity of metal oxide nanoparticles on two crop plants, maize (Zea mays $\mathrm{L}$.) and rice (Oryza sativa $\mathrm{L}$.). Int $\mathrm{J}$ Environ Res Public Health 12:15100-15109

Zhang L, Ding Y, Povey M, York D (2008) ZnO nanofluids—a potential antibacterial agent. Prog Nat Sci 18:939-944

Zhang Y, Ram MK, Stefanakos EK, Goswami DY (2012) Synthesis characterization, and applications of ZnO nanowires. J Nanomater 2012:1-22

Zhang D, Hua T, Xiao F, Chen C, Gersberg RM, Liu Y, Stuckey D, Ng WJ, Tan SK (2015) Phytotoxicity and bioaccumulation of $\mathrm{ZnO}$ nanoparticles in Schoenoplectus tabernaemontani. Chemosphere 120:211

Zhao J, Peralta-Videa JR, Ren M, Varela-Ramirez A, Li C, Hernandez-Viezcas JA, Aguilera RJ, Gardea-Torresdey JL (2012) Transport of Zn in a sandy loam soil treated with ZnO NPs and uptake by corn plants: Electron microprobe and confocal microscopy studies. Chem Eng J 184:1-8

Zhao L, Sun Y, Hernandez-Viezcas JA, Hong J, Majumdar S, Niu G, Duarte-Gardea M, PeraltaVidea JR, Gardea-Torresdey JL (2015) Monitoring the environmental effects of $\mathrm{CeO}_{2}$ and $\mathrm{ZnO}$ nanoparticles through the life cycle of corn (Zea mays) plants and in situ $\mu$-XRF mapping of nutrients in kernels. Environ Sci Technol 49:2921-2928

Zhou G, Li Y, Xiao W, Zhang L, Zuo Y, Xue J, Jansen JA (2008) Synthesis, characterization, and antibacterial activities of a novel nanohydroxyapatite/zinc oxide complex. J Biomed Mater Res A 85:929-937 